\title{
Novel Simplified Method for Coalbed Methane Feasibility Evaluation
}

\author{
Jose A. Gutiérrez, Raquel García, Erasmo Cadena \\ Department of Mining Engineering, University of León, Leon, Spain \\ Email: jagutbr@unileon.es
}

How to cite this paper: Gutiérrez, J.A., García, R. and Cadena, E. (2018) Novel Simplified Method for Coalbed Methane Feasibility Evaluation. International Journal of Clean Coal and Energy, 7, 59-92. https://doi.org/10.4236/ijcce.2018.74004

Received: October 22, 2018

Accepted: November 25, 2018

Published: November 28, 2018

Copyright $\odot 2018$ by authors and Scientific Research Publishing Inc. This work is licensed under the Creative Commons Attribution International License (CC BY 4.0).

http://creativecommons.org/licenses/by/4.0/

\section{(c) (i) Open Access}

\begin{abstract}
Coal during its carbonization process produces a gas. This gas, mainly formed by methane, can be used. This gas, coalbed methane (CBM), is usually mainly formed by methane and can be considered similar to natural gas as energy source. The evaluation of the techno-economic feasibility of the extraction of this gas depends on a large number of complex different factors. The work carried out covers the different aspects to simplify the first approach for CBM feasibility quantification considering a short number of indicators. A theoretical review and a state of the art description have been carried out, taking into account all the factors that can influence in the development of a CBM project. After that, technical feasibility has been used to evaluate total amount of gas that can be recovered. The last step was to evaluate economic feasibility to know how much gas could be economically profitable. Conclusions got have been used to develop a simple method for evaluating CBM economic feasibility considering just four easy known parameters of coal beds. These are: the rank, the thickness, the gas concentration, and the permeability.
\end{abstract}

\section{Keywords}

CBM, Feasibility Assessments, Coal, Natural Gas

\section{Introduction}

The gas contained in coal layers is an important energy resource capable of supporting the growing increase in energy demand. As unconventional deposits of natural gas, in addition to the coal layers, we can find natural gas in shales and low permeability sandstones. In this paper, we will focus only on the methane content of coal bed methane (CBM).

The first signs of the energy use of coal date back to $200 \mathrm{BC}$, according to Chinese records. In our days, and on a world scale, coal stands as the second type of 
fuel in order of consumption, although in our country its energy relevance has decreased in recent years. Due to the increase in coal consumption in Asia mainly, it is expected to become the first source of energy worldwide in the coming years.

The gas contained in the coal is a byproduct of the process of maturing it, as will be detailed later, which can be increased later due to different mechanisms. Initially, this gas was considered dangerous, so it was to reduce its concentration to increase safety in the mines. At present, this vision has changed and, far from diluting it, the maximum use of its energy potential is pursued.

The feasibility assessments for CBM exploitation cover a large number of complex of factors usually not available from the data sources of every coal basin. There is a need of performing a first approach of the economic feasibility of CBM exploitation with a simplified method.

This work is focused on the economic viability of the CBM production with the aim of knowing the possible development and factors influencing it. Several tables are proposed as a decision support system for CBM feasibility assessments.

The final use of these tables and factors will facilitate the first approach form of the economic feasibility of CBM extraction to carry out future detailed analysis of a coal basin.

\section{Types of Methane Emissions from Coal}

We have different origins of methane in coal layer, CBM (coal bed methane), which we can differentiate as follows:

- Methane from active mines, CMM (coal mine methane). It can also be obtained from ventilation air, VAM (ventilation air methane)

- Extraction of methane from abandoned mines, AMM (abandoned mine methane)

- Progress of surface surveys before the underground exploitation of coal. If the coal has not been extracted we would speak of methane in virgin carbon layer, VCBM (virgin coalbed methane).

- ECBM (enhanced coalbed methane), where the recovery of VCBM is stimulated by the injection of $\mathrm{N}_{2}$ or $\mathrm{CO}_{2}$. This method can be combined with the storage of $\mathrm{CO}_{2}$.

The following Table 1 shows the methane concentration most commonly obtained by each method and the gas flow in each case.

Table 1. Typical concentration of CBM systems [1].

\begin{tabular}{ccc}
\hline CBM source & Methane concentration (\%) & Gas flow $\left(\times 1000 \mathrm{~m}^{3} /\right.$ day $)$ \\
\hline VCBM & $>95$ & $1-18$ \\
CMM & $35-75$ & $6-195$ \\
VAM & 0.08 & $4-140$ \\
AMM & $35-90$ & $11-86$
\end{tabular}




\section{Methane in Virgin Carbon Layers (VCBM)}

The conventional exploitation of the VCBM is carried out by vertical drilling and hydraulic fracturing that helps to desorb the methane contained in the coal, although methods based on horizontal drilling are currently being successfully tested, thus recovering the VCBM more profitably.

The viability of a project of this type varies from 4 to $8 \mathrm{~m}^{3}$ of $\mathrm{CH}_{4} / \mathrm{t}$ of coal. The fundamental problems are the low permeability of coal, for which the layer is stimulated by various methods. This procedure requires a high number of surveys, which increases the operating costs.

Most coals have a porosity saturated in water. If a reduction of this pressure is caused by extracting the water by pumping, the methane of the micropores begins to desorb, which diffuses slowly through the fissures until reaching the sounding by which the depression has been caused.

The countries with the greatest potential for VCBM would be Russia, Canada, China, Australia and the United States. Even though the VCBM is a great source of clean energy, only the United States and Australia have well-established commercial productions [1].

\section{Potential CBM of Coal Layers}

For the methane in the coal layers to be an economically exploitable resource, the coal must present a series of characteristics that have been demonstrated after the experience acquired in the wells that are already in operation. It is useless to have a very powerful layer of coal if it has not produced the necessary gas or has not been able to store it.

Many times the exploration strategies are based on the location of the highest accumulated height, ignoring the interrelation between the geological and hydrogeological factors that affect the productivity of the $\mathrm{MBC}$, methodological neglect that leads to great failures in the exploration.

The MBC productivity of a coal deposit is determined by six closely interrelated factors:

- Tectonic and structural framework.

- Depositional framework and coal distribution.

- Rank and quality of coal.

- Gas content.

- Hydrodynamics.

- Permeability.

The fairway is the synergy of several factors that originate the areas with the highest gas content. They are characterized by:

- Groundwater flow towards the center of the basin.

- Generation of secondary biogenic gas.

- High coal rank.

- High concentration of gas towards flow barriers, which would cause an upward flow to discharge areas.

- Conventional gas entrapment migrated or in solution. 


\section{Methodology for Determining Each Parameter}

The objective of this chapter is to establish initial parameters of the reserve in order to know its production. CBM production depends on several parameters. In this section we will detail the methodology used to determine them.

A methodology for the initial estimation of CBM resources and reserves easily extrapolated has been developed. This methodology will serve to determine in what amount the parameters of the reserve should vary to obtain the return on investment. The calculations have been made in Anglo-Saxon units, due to their greater development in calculation methods and they have been converted to units of the international system later for a better compression. It will consist on the determination of:

- Coal saturation.

- Absolute permeability.

- Porosity, compressibility and pore volume

- Calculation of gas production.

\subsection{Coal Saturation}

Once the drilling point has been defined, the immediate analysis of the coal is taken and its rank determined. The analysis is corrected in ash, for an ash content of $15 \%$, which is the estimated content in the layer. The analysis of ash-free dry coal, which will be used in later calculations, is also corrected.

Once this is done, the gas content of the target layer is extracted and expressed both in gross ton, tb, (15\% ash) and per dry ton free of ash or pure ton (daf or tp).

Knowing the depth and the coal rank are known, we can estimate the maximum theoretical gas amount from the type adsorption isotherms of the Eddy diagram, Figure 1. By comparing the maximum capacity of adsorption with the gas content, we can know the degree of saturation of the coal.

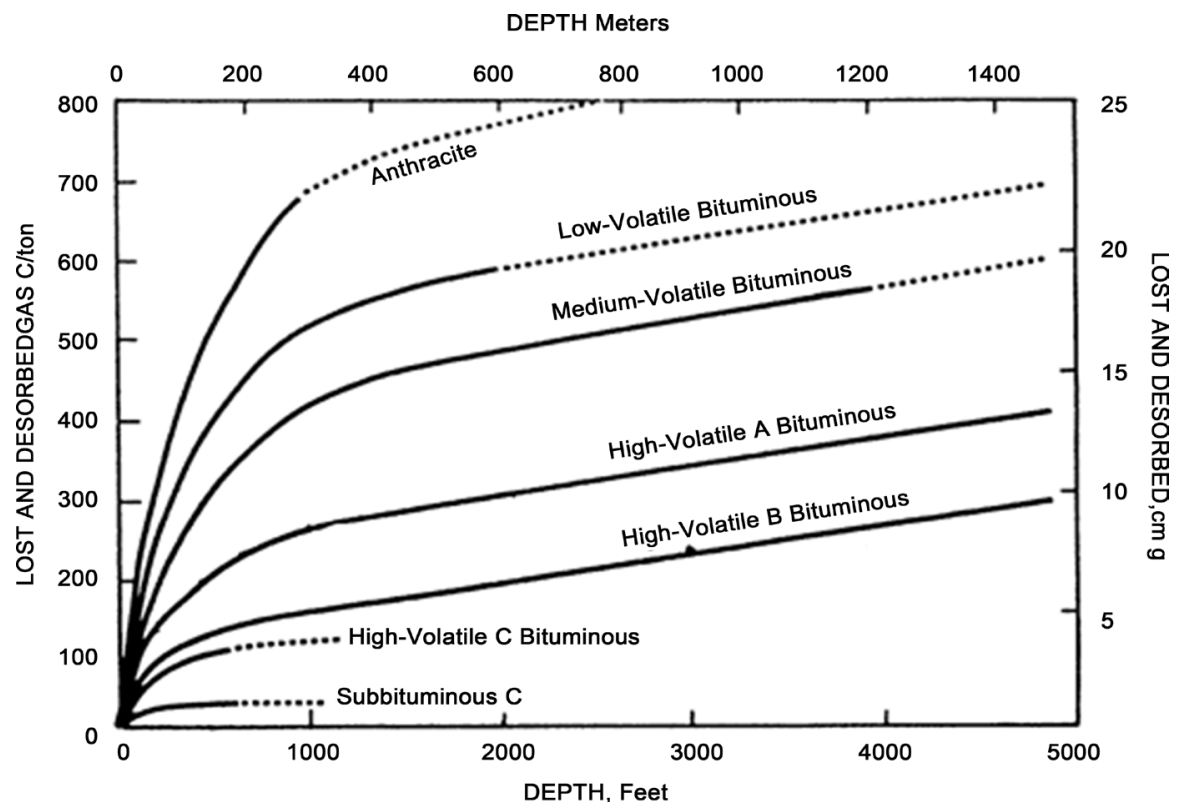

Figure 1. Eddy diagram for maximum gas adsorption [2]. 


\subsection{Calculation of Maximum Desorbed or Recovered Gas}

The next stage determines the calculation of the initial pressure of the matrix and of the fractures. The initial pressure of the fractures is established based on the depth, the initial pressure of the matrix is related to this. From the Eddy curve, a minimum desorption pressure is estimated, which will be the pressure of the fractures at which the coal will begin to desorb gas.

The final pressure of the well, and therefore of the fractures, is set at 75 psi as an operating parameter. Again entering the Eddy curve we can know the amount of gas not desorbed, and by difference with this, the percentage of gas recovered maximum.

\subsection{Absolute Permeability}

To determine the absolute permeability we will use the equation of Gray [3] that relates cleats and permeability:

$$
K=\frac{\left(1.013 \times 10^{10}\right) \times b^{3}}{12 \times s}
$$

Equation (1): Gray's Equation. Where:

- $b$ : width of cleats $(\mathrm{mm})$

- $s$ spacing of cleats $(\mathrm{mm})$

- $K$ : permeability $(\mathrm{mD})$

In order to determine the characteristics of the cleats, the following tables and graphs will be used, as well as articles about the spacing of cleats in the coal [4] [5] [6] and they are contrasted with data from other similar carbons and with results from various research articles on char properties. Table 2 shows cleats spacing depending on coal rank. Figure 2 shows cleats spacing regarding vitrinite reflectance and Figure 2.

In Figure 2 can be seen the importance of number of cleats and cleats aperture on the permeability of coal. Permeability and its evolution during CBM exploitation are crucial factors in the feasibility of a CBM exploitation. This permeability is mainly depending on cleat spacing, and aperture.

\subsection{Porosity, Compressibility and Pore volume}

The porosity will be estimated according to the range based on the analysis of the Gas Research Institute [7].

\subsection{Well Spacing}

Well spacing is manifested as a key factor for the economic viability of the reserve.

Table 2. Spacing of cleats depending on coal rank [5].

\begin{tabular}{cc}
\hline Coal rank & Cleats spacing $(\mathrm{cm})$ \\
\hline Sub-bituminous & $2-15$ \\
Bituminous high in volatiles & $0.36-2$ \\
Bituminous medium and low volatile & $<1$ \\
\hline
\end{tabular}




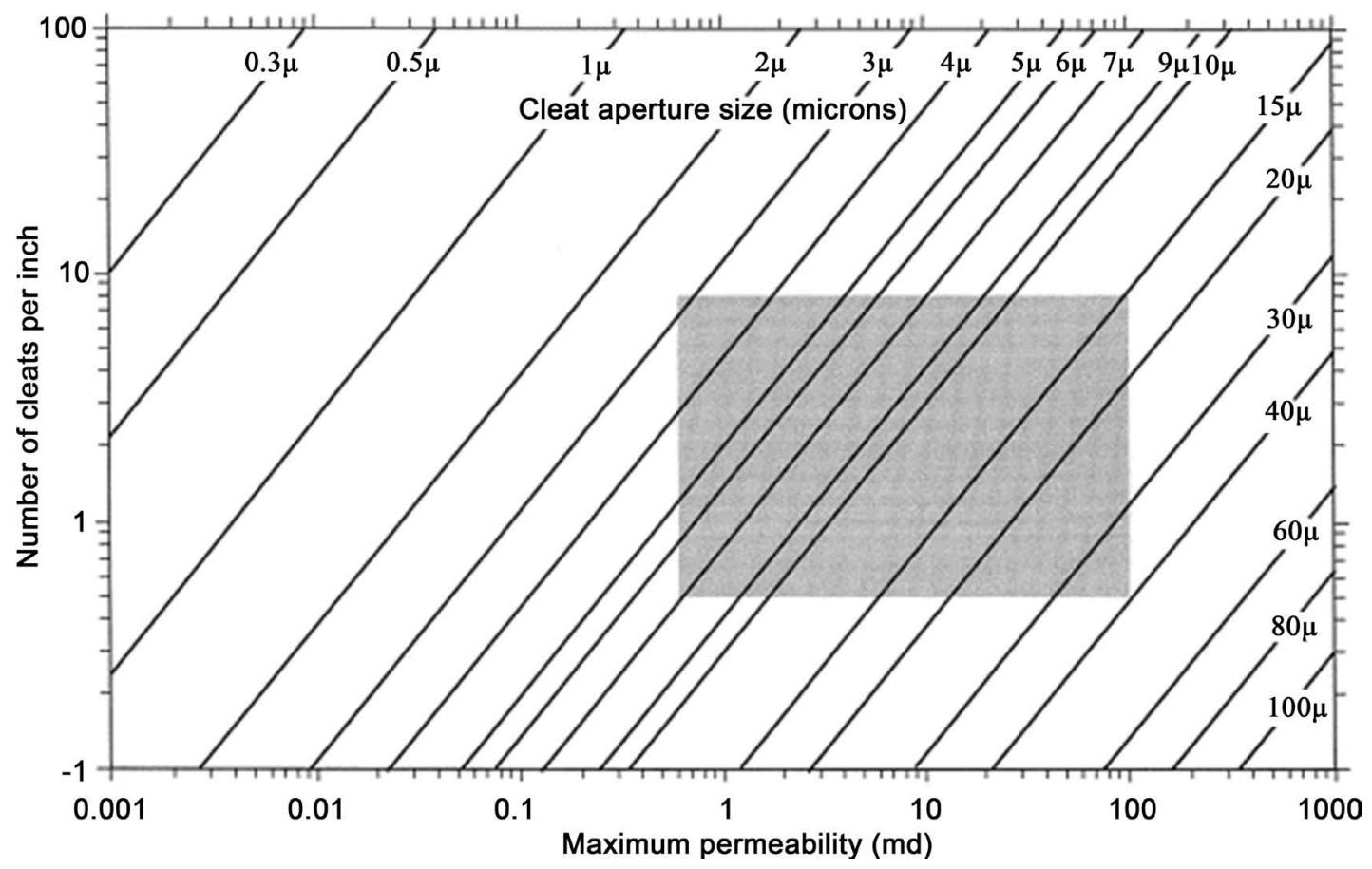

Figure 2. Relation permeability, cleats number and cleats spacing [4].

The well spacing is estimated according to the properties of the reserve, with permeability being the most important parameter. The upper and lower limits are set at 800 and $500 \mathrm{~m}$, or 120 and 60 acres respectively, surfaces that are considered appropriate for CBM exploitation.

\section{Estimation of Gas Production}

The estimation of gas production over time is carried out by developing the curves defined by García Arenas for the Department of Petroleum and Natural Gas Engineering of West Virginia [8]. They describe a curve model from two dimensionless parameters, $\mathrm{tD}$ and $\mathrm{qD}$, which thanks to their dimensionless condition are able to simulate the production of gas in any basin. Figure 3 shows the typical CBM production curve in logarithmic scale. The equations used are Equations (2)-(4).

This method has been proven by specific simulation software for CBM, such as the CMG GEM, with good results.

$$
q_{D}=\frac{q}{q_{\text {peak }}}
$$

Equation (2) Adimensional peak flow [8]

$$
t_{D}=\frac{t q_{\text {peak }}}{G_{i}}
$$

Equation (3) Adimensional time factor [8]

where:

- $q_{D}$ : dimensionless peak flow. 


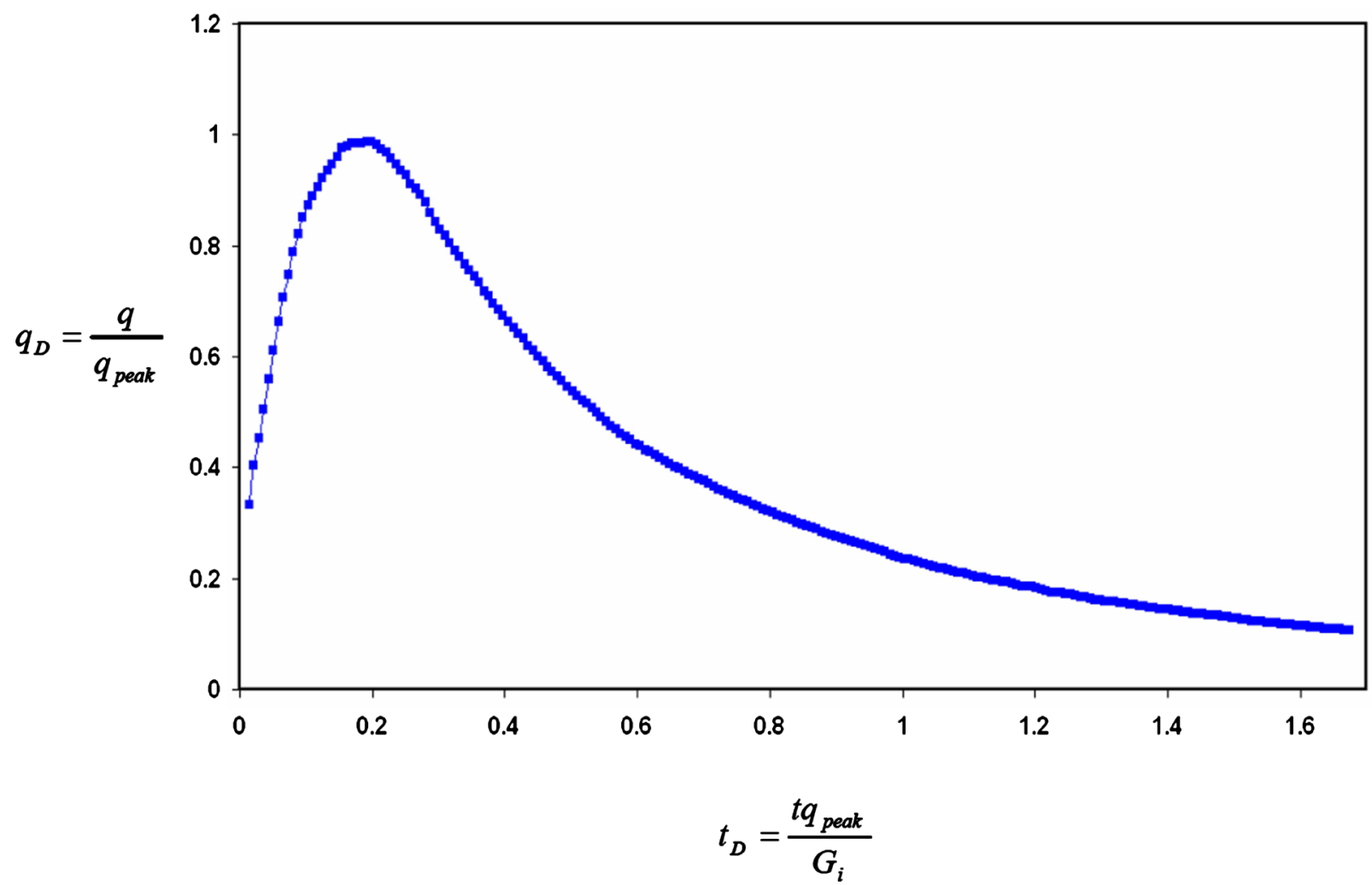

Figure 3. CBM production curve in logarithmic scale. In abscissas td, in ordinate qd [4].

- $Q$ : actual flow.

- $Q_{\text {peak }}$ peak or maximum flow.

- $t_{D}$ : dimensionless time factor.

- T: time in days.

- $G_{i}$ : amount of gas in place.

$G_{i}$ is determined multiplying coal quanty per coal gas contents. The greatest difficulty lies in the estimation of peak flow. It will come de-terminated by:

$$
q_{\text {peak }}=q D \cdot k \cdot h \cdot\left(P_{m}-P_{w f}\right)
$$

Equation (4) Peak flow [8]

where:

- $k$ permeability.

- $h$ : height of coal layer.

- $P_{m}$ : initial pressure of the matrix.

- $P_{w \dot{r}}$ initial pressure in the fracture system.

We have all the data of previous stages except the $q_{\text {peak }}$, which is obtained through the following Figure 4.

\section{Economic Feasibility}

In this section we will study the economic viability of CBM wells, in order to be able to decide on their exploitation. Once described, the development costs of a well type CBM will be defined for the selected cases. Later, the paths for the 


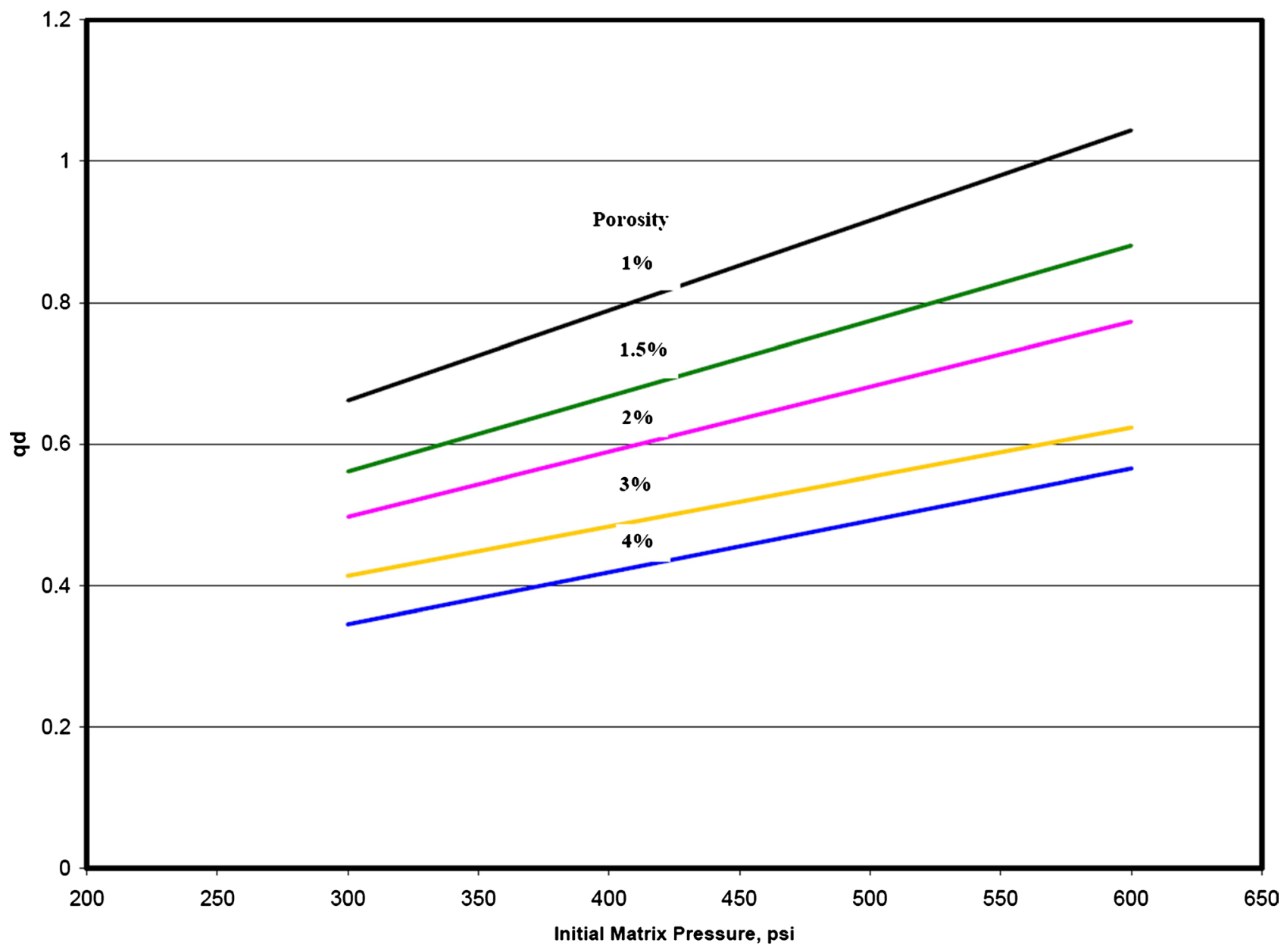

Figure 4. Dimensionless peak flow [8].

recovery of the investment will be defined.

The CBM extraction has been considered with own funds, without any other type of financing or state, banking, European subsidy, etc. The main risk factors in the development of a project of this type are: the capacity of the gas pipeline or the consumption to be obtained, the prices of natural gas, the sale price of the producer's gas and technological knowledge.

Three returns of investment will be defined, starting with the three of own funds. The first one will be an absolute return, for which the well covers the expenses generated by the gas generated, the second will be the one that gives us more profitability than an investment of $4 \%$ per year, and the third will also include the uncertainty of the investment that is considered at $8 \%$. These returns will be calculated for four scenarios of variation in the price of natural gas.

As a last step, the development of a method has been reached so that, based on simple parameters of the gas deposit, it is possible to quickly determine the viability of the CBM extraction.

\subsection{Costs of a CBM Well}

In this section, we will define the annual costs of a CBM well. Up to 20 years. 
The study conducted by the US.DOE [9] will be used as a basis. This is shown in Table 3.

From the reference of the drilling costs for 500 and 900 feet respectively, we can obtain the costs of our type drilling of $1000 \mathrm{~m}$. The capital investment costs (CAPEX) for the well would be shown in Table 4

The drilling and development costs of the surface installation for a CBM well are set at 240,000 euros. Regarding the cost of operation and maintenance, the US.DOE found the following values, shown in Table 5.

As annual fixed costs we can determine the following, shown in Table 6.

The variable costs that we can associate correspond to the capture and storage

Table 3. Drilling costs CBM [9].

\begin{tabular}{cccc}
\hline Depth $(\mathrm{ft})$ & 500 & 950 & \\
Depth $(\mathrm{m})$ & 152 & 290 & 1.000 \\
Dilling costs $(€)$ & $\mathbf{6 0 . 0 0 0}$ & $\mathbf{7 4 . 0 0 0}$ & $\mathbf{1 4 6 . 5 1 5}$ \\
Intangibles & 50.000 & 62.000 & \\
Tangibles & 10.000 & 12.000 & \\
Well completion costs $(€)$ & $\mathbf{2 2 . 5 0 0}$ & $\mathbf{2 7 . 7 5 0}$ & $\mathbf{5 4 . 9 4 3}$ \\
Intangibles & 7.500 & 9.250 & \\
Tangibles & 15.000 & 18.500 & \\
\hline
\end{tabular}

Table 4. CAPEX costs of a CBM well [9].

\begin{tabular}{cc}
\hline Concept & Costs $(€)$ \\
\hline Permits & 12,000 \\
Drilling and finishing well $(1000 \mathrm{~m})$ & 160,000 \\
Water collection & 20,000 \\
Water removal & 1200 \\
Electric energy & 10,000 \\
Gas collection & 35,000 \\
Total & 238,200 \\
\hline
\end{tabular}

Table 5. O \& M costs of a CBM well [9].

\begin{tabular}{cc}
\hline Year & Costs $(€)$ \\
\hline 1 & 37,500 \\
$2-4$ & 20,000 \\
$<4$ & 15,000 \\
\hline
\end{tabular}

Table 6. Annual fixed costs of a CBM well [9].

\begin{tabular}{cccc}
\hline Leasing & Management & Water treatment & Maintenance \\
\hline 15,000 & 9000 & 3000 & 10,000 \\
\hline
\end{tabular}


of natural gas and its transportation. Capture and storage of NG is $€ 0.006 / \mathrm{m}^{3}$ Transportation is estimated in $€ 0.003 / \mathrm{Nm}^{3}$.

\subsection{Revenue from Extraction of the CBM}

The expected revenues will come in fully from the sales of the recovered gas. Although it is possible to obtain financing as described above, as well as the technology itself, these aids will depend on the body that develops the project, in this study are not going to take into account these income derived from the potential financing exposed.

Regarding the price of the CBM, for natural gas the price depends on the market. The market sets a calorific value of the gas at $34.48 \mathrm{MJ} / \mathrm{m}^{3}$, which will be used in the conversion. Due to the variability of the price of gas, revenues may fluctuate significantly, so a sensitivity analysis of the feasibility of the project is carried out, with four possible scenarios based on this price variability of the gas. At first, the price of gas remained constant. The second would contemplate a gas price $25 \%$ lower than the current one. The third and fourth would be made considering an increase of $25 \%$ and $50 \%$ of the price of gas respectively, this is shown in Table 7.

\section{Determination of Parameters for Viable Production}

As described before, there are several parameters that condition the technical and economic viability of the development of a CBM project. Once analyzed the results on the technical and economic viability it is possible not only to know factors that affect the viable production, but also they can be quantified.

In this section we will quantify the minimum factors for viable production. It has been possible to develop a method to reduce the number of parameters and from it, to be able to determine the economic viability of the reserve. They are specified as:

- Geometry of the reserve. Surface area and height.

- Coal rank.

- Gas in situ or amount of gas.

- Permeability.

Based on these four parameters we should be able to know the possibility of extracting CBM.

The minimum amount of gas in situ was not found as relevant as initially

Table 7. Gas price scenarios.

\begin{tabular}{cccc}
\hline & Price NG & $€ / \mathrm{MWh}$ & $€ / \mathrm{m}^{3}$ \\
\hline Scenario 1 & Reference & 26.413 & 0.2527 \\
Scenario 2 & $-25 \%$ & 21.1304 & 0.2022 \\
Scenario 3 & $+25 \%$ & 33.01625 & 0.3159 \\
Scenario 4 & $+50 \%$ & 39.6195 & 0.3791 \\
\hline
\end{tabular}


expected. The minimum quantity depends exclusively on the coal rank, varying from $12 \mathrm{~m}^{3} / \mathrm{t}$ pure in anthracites to $5 \mathrm{~m}^{3} / \mathrm{t}$ pure in high bituminous carbons in volatile type $\mathrm{B}$. The rest of the minimum values of gas in situ can be seen in the following Table 8.

The rest of factors will depend on the profitability that you want to obtain in the project, so the data will be analyzed according to this objective. Thus, for a positive return (IRR $>0 \%$ ) we will obtain the same minimum values as for a return greater than $4 \%$ or $8 \%$. First let's analyze the minimum values for positive profitable production

\subsection{Positive Profitable Production (IRR $>0 \%$ )}

The average annual production $\left(\mathrm{m}^{3}\right)$ required for minimum economic profitability (IRR $>0 \%$ ) depends on the operation time of the well and the gas prices. The following Table 9 shows the minimum annual average production values for minimum profitable production. We observe how this minimum annual average production decreases with the operating time. In addition, you can check the influence of the price of gas.

Depending on the gas price scenario, the amount of gas to drain annually will vary. This amount will depend on the permeability and the height of the layer. The following Table 10 gives the minimum height for several permeability values in each of the scenarios. It can be verified that, when the permeability is low, the minimum height required increases, while as the permeability increases, the minimum height of the layer is reduced very significantly.

\subsection{Cost-Effective Production with Higher Profitability (IRR $>4 \%$ )}

Considering that own funds can yield up to $4 \%$ in a fixed term, this percentage is set at least for viable production. With which the previous average annual

Table 8. Minimum gas concentration for profitability according to the range.

\begin{tabular}{cc}
\hline Coal Rank & Gas concentration $\left(\mathrm{m}^{3} / \mathrm{t}\right.$ pure $)$ \\
\hline Anthracite & 12 \\
Bituminous low volatile & 8 \\
Bituminous medium in volatile & 7 \\
Bituminous high in volatile type A & 6 \\
Bituminous high in volatile type B & 5
\end{tabular}

Table 9. Average annual minimum production for positive profitability $\left(\mathrm{m}^{3}\right)$.

\begin{tabular}{ccccc}
\hline Years & Scenario 1 & Scenario 2 & Scenario 3 & Scenario 4 \\
\hline $\mathbf{1 0}$ & 325,000 & 410,000 & 260,000 & 215,000 \\
$\mathbf{2 0}$ & 270,000 & 340,000 & 215,000 & 180,000 \\
$\mathbf{3 0}$ & 250,000 & 315,000 & 200,000 & 170,000 \\
$\mathbf{5 0}$ & 245,000 & 305,000 & 195,000 & 160,000 \\
\hline
\end{tabular}


minimum production would be modified, as shown in Table 11 . The new values would be:

Depending on the gas price scenario, the amount to drain annually will vary. This amount will depend on the permeability and the height of the layer. The following Table 12 details the minimum height for several permeability values in each of the scenarios.

Table 10. Minimum height for various permeability values.

\begin{tabular}{ccccc}
\hline IRR $>$ 0\% & Scenario 1 & Scenario 2 & Scenario 3 & Scenario 4 \\
\hline $\begin{array}{c}\text { Permeability } \\
(\mathrm{mD})\end{array}$ & $\begin{array}{c}\text { Minimum height } \\
(\mathrm{m})\end{array}$ & $\begin{array}{c}\text { Minimum height } \\
(\mathrm{m})\end{array}$ & $\begin{array}{c}\text { Minimum height } \\
(\mathrm{m})\end{array}$ & $\begin{array}{c}\text { Minimum height } \\
(\mathrm{m})\end{array}$ \\
\hline $\mathbf{1}$ & 45 & 55 & 35 & 30 \\
$\mathbf{2 . 5}$ & 9.5 & 12 & 7.5 & 6.5 \\
$\mathbf{5}$ & 4 & 5 & 3 & 2.6 \\
7.5 & 2.6 & 3.2 & 2.2 & 1.8 \\
$>10$ & 2.1 & 2.6 & 1.7 & 1.4 \\
\hline
\end{tabular}

Table 11. Average annual minimum production for profitability greater than $4 \%\left(\mathrm{~m}^{3}\right)$.

\begin{tabular}{ccccc}
\hline Years & Scenario 1 & Scenario 2 & Scenario 3 & Scenario 4 \\
\hline $\mathbf{1 0}$ & 345,000 & 440,000 & 275,000 & 230,000 \\
$\mathbf{2 0}$ & 290,000 & 365,000 & 230,000 & 195,000 \\
$\mathbf{3 0}$ & 275,000 & 345,000 & 220,000 & 180,000 \\
$\mathbf{5 0}$ & 270,000 & 330,000 & 210,000 & 175,000 \\
\hline
\end{tabular}

Table 12. Minimum height for various permeability values.

\begin{tabular}{ccccc}
\hline IRR $>$ 4\% & Scenario 1 & Scenario 2 & Scenario 3 & Scenario 4 \\
\hline $\begin{array}{c}\text { Permeability } \\
(\mathrm{mD})\end{array}$ & $\begin{array}{c}\text { Minimum height } \\
(\mathrm{m})\end{array}$ & $\begin{array}{c}\text { Minimum height } \\
(\mathrm{m})\end{array}$ & $\begin{array}{c}\text { Minimum height } \\
(\mathrm{m})\end{array}$ & $\begin{array}{c}\text { Minimum height } \\
(\mathrm{m})\end{array}$ \\
\hline $\mathbf{1}$ & 1 & 50 & 60 & 40 \\
2.5 & 2.5 & 10 & 13 & 8.5 \\
5 & 5 & 4.5 & 5.5 & 3.3 \\
7.5 & 7.5 & 2.8 & 3.5 & 2.4 \\
$>10$ & $>10$ & 2.3 & 2.9 & 1.8 \\
\hline
\end{tabular}

\subsection{Profitable Production Higher Profitability (IRR > 8\%)}

Considering that own funds can yield up to $4 \%$ in a fixed term and the uncertainty of the investment is set at $8 \%$ the minimum percentage to make the project attractive. With which the previous average annual minimum production would be modified. The new values would be shown in Table 13 and Table 14 .

Depending on the gas price scenario, the amount to drain annually will vary. This amount will depend on the permeability and the height of the layer. The 
Table 13. Average annual minimum production for profitability greater than $8 \%\left(\mathrm{~m}^{3}\right)$.

\begin{tabular}{ccccc}
\hline Years & Scenario 1 & Scenario 2 & Scenario 3 & Scenario 4 \\
\hline $\mathbf{1 0}$ & 370.000 & 465.000 & 295.000 & 245.000 \\
$\mathbf{2 0}$ & 320.000 & 400.000 & 255.000 & 210.000 \\
$\mathbf{3 0}$ & 305.000 & 380.000 & 240.000 & 200.000 \\
$\mathbf{5 0}$ & 300.000 & 370.000 & 235.000 & 195.000 \\
\hline
\end{tabular}

Table 14. Minimum height for various permeability values.

\begin{tabular}{ccccc}
\hline IRR $>$ 4\% & Scenario 1 & Scenario 2 & Scenario 3 & Scenario 4 \\
\hline $\begin{array}{c}\text { Permeability } \\
(\mathrm{mD})\end{array}$ & $\begin{array}{c}\text { Minimum height } \\
(\mathrm{m})\end{array}$ & $\begin{array}{c}\text { Minimum height } \\
(\mathrm{m})\end{array}$ & $\begin{array}{c}\text { Minimum height } \\
(\mathrm{m})\end{array}$ & $\begin{array}{c}\text { Minimum height } \\
(\mathrm{m})\end{array}$ \\
\hline $\mathbf{1}$ & 53 & 66 & 42 & 35 \\
$\mathbf{2 . 5}$ & 12 & 13 & 9 & 7.5 \\
$\mathbf{5}$ & 4.6 & 5.7 & 3.6 & 3 \\
7.5 & 3.1 & 3.5 & 2.5 & 2.1 \\
$>\mathbf{1 0}$ & 2.5 & 3.1 & 2.0 & 1.7 \\
\hline
\end{tabular}

following table details the minimum height for several permeability values in each of the scenarios. It can be verified that, when the permeability is low, the minimum height required increases, while as the permeability increases, the minimum height of the layer is reduced very significantly as shown in Table 14.

\section{Minimum Basic Characteristics for Profitable Production}

By developing the above, tables can be obtained that will determine the minimum values for viable production. Next, these tables will be exposed in which several parameters are evaluated:

- IRR.

- Price of natural gas.

- Coal rank.

- Gas in place (GIP).

- Gas in situ (GIS) or amount of gas.

- Height of the bad.

- Permeability.

The input data would be the expected return or IRR, price of gas (scenario) and the rank of coal. In the cells corresponding to the other four factors: GIP, GIS, height and permeability, the minimum values for profitable production are determined, so that to obtain positive profitability all of them must be overcome.

A group of 24 tables, named as Table 15, have been developed, one for each profitability and price scenario of the gas valued, for 30 and 10 years of production respectively. Once the production time is set, 10 or 30 years, the first thing to look for in the tables will be the minimum profitability required: $0 \%, 4 \%$ or $8 \%$. Next, the gas price scenario will be assessed. The next step will be to determine 
Table 15. Minimum conditions for profitability (Group of tables).

\begin{tabular}{|c|c|c|c|c|c|c|}
\hline IRR & Price & Rank & GIP area min. & GIS min. & Height min. & Perm \\
\hline 30 years & $€ / \mathrm{m}^{3}$ & & $\mathrm{~m}^{3}$ & $\mathrm{~m}^{3} / \mathrm{tp}$ & $\mathrm{m}$ & $\mathrm{mD}$ \\
\hline IRR $>0 \%$ & Scenario 1 & Anthracite & $346,759,920$ & 12 & 45 & 1 \\
\hline IRR $>0 \%$ & Scenario 1 & Anthracite & $73,204,872$ & 12 & 9.5 & 2.5 \\
\hline IRR $>0 \%$ & Scenario 1 & Anthracite & $30,823,104$ & 12 & 4.0 & 5 \\
\hline IRR $>0 \%$ & Scenario 1 & Anthracite & $20,035,018$ & 12 & 2.6 & 7.5 \\
\hline IRR $>0 \%$ & Scenario 1 & Anthracite & $16,182,130$ & 12 & 2.1 & 10 \\
\hline IRR $>0 \%$ & Scenario 1 & Bituminous low volatiles & $231,173,280$ & 8 & 45 & 1 \\
\hline IRR $>0 \%$ & Scenario 1 & Bituminous low volatiles & $48,803,248$ & 8 & 9.5 & 2.5 \\
\hline IRR $>0 \%$ & Scenario 1 & Bituminous low volatiles & $20,548,736$ & 8 & 4.0 & 5 \\
\hline IRR $>0 \%$ & Scenario 1 & Bituminous low volatiles & $13,356,678$ & 8 & 2.6 & 7.5 \\
\hline IRR $>0 \%$ & Scenario 1 & Bituminous low volatiles & $10,788,086$ & 8 & 2.1 & 10 \\
\hline IRR $>0 \%$ & Scenario 1 & Bituminous medium volatiles & $202,276,620$ & 7 & 45 & 1 \\
\hline IRR $>0 \%$ & Scenario 1 & Bituminous medium volatiles & $42,702,842$ & 7 & 9.5 & 2.5 \\
\hline IRR $>0 \%$ & Scenario 1 & Bituminous medium volatiles & $17,980,144$ & 7 & 4.0 & 5 \\
\hline IRR $>0 \%$ & Scenario 1 & Bituminous medium volatiles & $11,687,094$ & 7 & 2.6 & 7.5 \\
\hline IRR $>0 \%$ & Scenario 1 & Bituminous medium volatiles & $9,439,576$ & 7 & 2.1 & 10 \\
\hline IRR $>0 \%$ & Scenario 1 & Bituminous high volatiles A & $173,379,960$ & 6 & 45 & 1 \\
\hline IRR $>0 \%$ & Scenario 1 & Bituminous high volatiles A & $36,602,436$ & 6 & 9.5 & 2.5 \\
\hline IRR $>0 \%$ & Scenario 1 & Bituminous high volatiles A & $15,411,552$ & 6 & 4.0 & 5 \\
\hline IRR $>0 \%$ & Scenario 1 & Bituminous high volatiles A & $10,017,509$ & 6 & 2.6 & 7.5 \\
\hline IRR $>0 \%$ & Scenario 1 & Bituminous high volatiles A & $8,091,065$ & 6 & 2.1 & 10 \\
\hline IRR $>0 \%$ & Scenario 1 & Bituminous high volatiles B & $144,483,300$ & 5 & 45 & 1 \\
\hline IRR $>0 \%$ & Scenario 1 & Bituminous high volatiles B & $30,502,030$ & 5 & 9.5 & 2.5 \\
\hline IRR $>0 \%$ & Scenario 1 & Bituminous high volatiles B & $12,842,960$ & 5 & 4.0 & 5 \\
\hline IRR $>0 \%$ & Scenario 1 & Bituminous high volatiles B & $8,347,924$ & 5 & 2.6 & 7.5 \\
\hline IRR $>0 \%$ & Scenario 1 & Bituminous high volatiles B & $6,742,554$ & 5 & 2.1 & 10 \\
\hline IRR & Price & Rank & GIP area min & GIS min. & Height min. & Perm \\
\hline 30 years & $€ / \mathrm{m}^{3}$ & & $\mathrm{~m}^{3}$ & $\mathrm{~m}^{3} / \mathrm{tp}$ & $\mathrm{m}$ & $\mathrm{mD}$ \\
\hline IRR $>0 \%$ & Scenario 2 & Anthracite & $423,817,680$ & 12 & 55 & 1 \\
\hline IRR $>0 \%$ & Scenario 2 & Anthracite & $92,469,312$ & 12 & 12.0 & 2.5 \\
\hline IRR $>0 \%$ & Scenario 2 & Anthracite & $38,528,880$ & 12 & 5.0 & 5 \\
\hline IRR $>0 \%$ & Scenario 2 & Anthracite & $24,658,483$ & 12 & 3.2 & 7.5 \\
\hline IRR $>0 \%$ & Scenario 2 & Anthracite & $20,035,018$ & 12 & 2.6 & 10 \\
\hline IRR $>0 \%$ & Scenario 2 & Bituminous low volatiles & $282,545,120$ & 8 & 55 & 1 \\
\hline IRR $>0 \%$ & Scenario 2 & Bituminous low volatiles & $61,646,208$ & 8 & 12.0 & 2.5 \\
\hline IRR $>0 \%$ & Scenario 2 & Bituminous low volatiles & $25,685,920$ & 8 & 5.0 & 5 \\
\hline
\end{tabular}




\section{Continued}

\begin{tabular}{|c|c|c|c|c|c|c|}
\hline $\mathrm{IRR}>0 \%$ & Scenario 2 & Bituminous low volatiles & $16,438,989$ & 8 & 3.2 & 7.5 \\
\hline IRR $>0 \%$ & Scenario 2 & Bituminous low volatiles & $13,356,678$ & 8 & 2.6 & 10 \\
\hline $\mathrm{IRR}>0 \%$ & Scenario 2 & Bituminous medium volatiles & $247,226,980$ & 7 & 55 & 1 \\
\hline IRR $>0 \%$ & Scenario 2 & Bituminous medium volatiles & $53,940,432$ & 7 & 12.0 & 2.5 \\
\hline $\mathrm{IRR}>0 \%$ & Scenario 2 & Bituminous medium volatiles & $22,475,180$ & 7 & 5.0 & 5 \\
\hline $\mathrm{IRR}>0 \%$ & Scenario 2 & Bituminous medium volatiles & $14,384,115$ & 7 & 3.2 & 7.5 \\
\hline IRR $>0 \%$ & Scenario 2 & Bituminous medium volatiles & $11,687,094$ & 7 & 2.6 & 10 \\
\hline $\mathrm{IRR}>0 \%$ & Scenario 2 & Bituminous high volatiles A & $211,908,840$ & 6 & 55 & 1 \\
\hline IRR $>0 \%$ & Scenario 2 & Bituminous high volatiles A & $46,234,656$ & 6 & 12.0 & 2.5 \\
\hline $\mathrm{IRR}>0 \%$ & Scenario 2 & Bituminous high volatiles A & $19,264,440$ & 6 & 5.0 & 5 \\
\hline $\mathrm{IRR}>0 \%$ & Scenario 2 & Bituminous high volatiles A & $12,329,242$ & 6 & 3.2 & 7.5 \\
\hline $\mathrm{IRR}>0 \%$ & Scenario 2 & Bituminous high volatiles A & $10,017,509$ & 6 & 2.6 & 10 \\
\hline $\mathrm{IRR}>0 \%$ & Scenario 2 & Bituminous high volatiles B & $176,590,700$ & 5 & 55 & 1 \\
\hline IRR $>0 \%$ & Scenario 2 & Bituminous high volatiles B & $38,528,880$ & 5 & 12.0 & 2.5 \\
\hline $\mathrm{IRR}>0 \%$ & Scenario 2 & Bituminous high volatiles B & $16,053,700$ & 5 & 5.0 & 5 \\
\hline $\mathrm{IRR}>0 \%$ & Scenario 2 & Bituminous high volatiles B & $10,274,368$ & 5 & 3.2 & 7.5 \\
\hline $\mathrm{IRR}>0 \%$ & Scenario 2 & Bituminous high volatiles B & $8,347,924$ & 5 & 2.6 & 10 \\
\hline IRR & Price & Rank & GIP area min & GIS min. & Height min. & Perm \\
\hline 30 years & $€ / \mathrm{m}^{3}$ & & $\mathrm{~m}^{3}$ & $\mathrm{~m}^{3} / \mathrm{tp}$ & m & $\mathrm{mD}$ \\
\hline $\mathrm{IRR}>0 \%$ & Scenario 3 & Anthracite & $269,702,160$ & 12 & 35 & 1 \\
\hline $\mathrm{IRR}>0 \%$ & Scenario 3 & Anthracite & $57,793,320$ & 12 & 7.5 & 2.5 \\
\hline $\mathrm{IRR}>0 \%$ & Scenario 3 & Anthracite & $23,117,328$ & 12 & 3.0 & 5 \\
\hline IRR $>0 \%$ & Scenario 3 & Anthracite & $16,952,707$ & 12 & 2.2 & 7.5 \\
\hline IRR $>0 \%$ & Scenario 3 & Anthracite & $13,099,819$ & 12 & 1.7 & 10 \\
\hline $\mathrm{IRR}>0 \%$ & Scenario 3 & Bituminous low volatiles & $179,801,440$ & 8 & 35 & 1 \\
\hline IRR $>0 \%$ & Scenario 3 & Bituminous low volatiles & $38,528,880$ & 8 & 7.5 & 2.5 \\
\hline $\mathrm{IRR}>0 \%$ & Scenario 3 & Bituminous low volatiles & $15,411,552$ & 8 & 3.0 & 5 \\
\hline IRR $>0 \%$ & Scenario 3 & Bituminous low volatiles & $11,301,805$ & 8 & 2.2 & 7.5 \\
\hline $\mathrm{IRR}>0 \%$ & Scenario 3 & Bituminous low volatiles & $8,733,213$ & 8 & 1.7 & 10 \\
\hline $\mathrm{IRR}>0 \%$ & Scenario 3 & Bituminous medium volatiles & $157,326,260$ & 7 & 35 & 1 \\
\hline $\mathrm{IRR}>0 \%$ & Scenario 3 & Bituminous medium volatiles & $33,712,770$ & 7 & 7.5 & 2.5 \\
\hline $\mathrm{IRR}>0 \%$ & Scenario 3 & Bituminous medium volatiles & $13,485,108$ & 7 & 3.0 & 5 \\
\hline IRR $>0 \%$ & Scenario 3 & Bituminous medium volatiles & $9,889,079$ & 7 & 2.2 & 7.5 \\
\hline $\mathrm{IRR}>0 \%$ & Scenario 3 & Bituminous medium volatiles & $7,641,561$ & 7 & 1.7 & 10 \\
\hline IRR $>0 \%$ & Scenario 3 & Bituminous high volatiles A & $134,851,080$ & 6 & 35 & 1 \\
\hline $\mathrm{IRR}>0 \%$ & Scenario 3 & Bituminous high volatiles A & $28,896,660$ & 6 & 7.5 & 2.5 \\
\hline IRR $>0 \%$ & Scenario 3 & Bituminous high volatiles A & $11,558,664$ & 6 & 3.0 & 5 \\
\hline
\end{tabular}




\section{Continued}

\begin{tabular}{|c|c|c|c|c|c|c|}
\hline IRR $>0 \%$ & Scenario 3 & Bituminous high volatiles A & $8,476,354$ & 6 & 2.2 & 7.5 \\
\hline IRR $>0 \%$ & Scenario 3 & Bituminous high volatiles $\mathrm{A}$ & $6,549,910$ & 6 & 1.7 & 10 \\
\hline IRR $>0 \%$ & Scenario 3 & Bituminous high volatiles $B$ & $112,375,900$ & 5 & 35 & 1 \\
\hline IRR $>0 \%$ & Scenario 3 & Bituminous high volatiles B & $24,080,550$ & 5 & 7.5 & 2.5 \\
\hline IRR $>0 \%$ & Scenario 3 & Bituminous high volatiles $B$ & $9,632,220$ & 5 & 3.0 & 5 \\
\hline IRR $>0 \%$ & Scenario 3 & Bituminous high volatiles B & $7,063,628$ & 5 & 2.2 & 7.5 \\
\hline IRR $>0 \%$ & Scenario 3 & Bituminous high volatiles $B$ & $5,458,258$ & 5 & 1.7 & 10 \\
\hline IRR & Price & Rank & GIP area min & GIS min. & Height min. & Perm \\
\hline 30 years & $€ / \mathrm{m}^{3}$ & & $\mathrm{~m}^{3}$ & $\mathrm{~m}^{3} / \mathrm{tp}$ & $\mathrm{m}$ & $\mathrm{mD}$ \\
\hline IRR $>0 \%$ & Scenario 4 & Anthracite & $231,173,280$ & 12 & 30 & 1 \\
\hline IRR $>0 \%$ & Scenario 4 & Anthracite & $50,087,544$ & 12 & 6.5 & 2.5 \\
\hline IRR $>0 \%$ & Scenario 4 & Anthracite & $20,035,018$ & 12 & 2.6 & 5 \\
\hline IRR $>0 \%$ & Scenario 4 & Anthracite & $13,870,397$ & 12 & 1.8 & 7.5 \\
\hline IRR $>0 \%$ & Scenario 4 & Anthracite & $10,788,086$ & 12 & 1.4 & 10 \\
\hline IRR $>0 \%$ & Scenario 4 & Bituminous low volatiles & $154,115,520$ & 8 & 30 & 1 \\
\hline IRR $>0 \%$ & Scenario 4 & Bituminous low volatiles & $33,391,696$ & 8 & 6.5 & 2.5 \\
\hline IRR $>0 \%$ & Scenario 4 & Bituminous low volatiles & $13,356,678$ & 8 & 2.6 & 5 \\
\hline IRR $>0 \%$ & Scenario 4 & Bituminous low volatiles & $9,246,931$ & 8 & 1.8 & 7.5 \\
\hline IRR $>0 \%$ & Scenario 4 & Bituminous low volatiles & $7,192,058$ & 8 & 1.4 & 10 \\
\hline IRR $>0 \%$ & Scenario 4 & Bituminous medium volatiles & $134,851,080$ & 7 & 30 & 1 \\
\hline IRR $>0 \%$ & Scenario 4 & Bituminous medium volatiles & $29,217,734$ & 7 & 6.5 & 2.5 \\
\hline IRR $>0 \%$ & Scenario 4 & Bituminous medium volatiles & $11,687,094$ & 7 & 2.6 & 5 \\
\hline IRR $>0 \%$ & Scenario 4 & Bituminous medium volatiles & $8,091,065$ & 7 & 1.8 & 7.5 \\
\hline IRR $>0 \%$ & Scenario 4 & Bituminous medium volatiles & $6,293,050$ & 7 & 1.4 & 10 \\
\hline IRR $>0 \%$ & Scenario 4 & Bituminous high volatiles A & $115,586,640$ & 6 & 30 & 1 \\
\hline IRR $>0 \%$ & Scenario 4 & Bituminous high volatiles A & $25,043,772$ & 6 & 6.5 & 2.5 \\
\hline IRR $>0 \%$ & Scenario 4 & Bituminous high volatiles A & $10,017,509$ & 6 & 2.6 & 5 \\
\hline IRR $>0 \%$ & Scenario 4 & Bituminous high volatiles A & $6,935,198$ & 6 & 1.8 & 7.5 \\
\hline IRR $>0 \%$ & Scenario 4 & Bituminous high volatiles A & $5,394,043$ & 6 & 1.4 & 10 \\
\hline IRR $>0 \%$ & Scenario 4 & Bituminous high volatiles B & $96,322,200$ & 5 & 30 & 1 \\
\hline IRR $>0 \%$ & Scenario 4 & Bituminous high volatiles $B$ & $20,869,810$ & 5 & 6.5 & 2.5 \\
\hline IRR $>0 \%$ & Scenario 4 & Bituminous high volatiles B & $8,347,924$ & 5 & 2.6 & 5 \\
\hline IRR $>0 \%$ & Scenario 4 & Bituminous high volatiles B & $5,779,332$ & 5 & 1.8 & 7.5 \\
\hline IRR $>0 \%$ & Scenario 4 & Bituminous high volatiles B & $4,495,036$ & 5 & 1.4 & 10 \\
\hline IRR & Price & Rank & GIP area min & GIS min. & Height min. & Perm \\
\hline 30 years & $€ / \mathrm{m}^{3}$ & & $\mathrm{~m}^{3}$ & $\mathrm{~m}^{3} / \mathrm{tp}$ & $\mathrm{m}$ & $\mathrm{mD}$ \\
\hline IRR $>4 \%$ & Scenario 1 & Anthracite & $385,288,800$ & 12 & 50 & 1 \\
\hline
\end{tabular}




\section{Continued}

\begin{tabular}{|c|c|c|c|c|c|c|}
\hline $\mathrm{IRR}>4 \%$ & Scenario 1 & Anthracite & $80,910,648$ & 12 & 10.5 & 2.5 \\
\hline IRR $>4 \%$ & Scenario 1 & Anthracite & $34,675,992$ & 12 & 4.5 & 5 \\
\hline $\mathrm{IRR}>4 \%$ & Scenario 1 & Anthracite & $21,576,173$ & 12 & 2.8 & 7.5 \\
\hline $\mathrm{IRR}>4 \%$ & Scenario 1 & Anthracite & $17,723,285$ & 12 & 2.3 & 10 \\
\hline $\mathrm{IRR}>4 \%$ & Scenario 1 & Bituminous low volatiles & $256,859,200$ & 8 & 50 & 1 \\
\hline $\mathrm{IRR}>4 \%$ & Scenario 1 & Bituminous low volatiles & $53,940,432$ & 8 & 10.5 & 2.5 \\
\hline $\mathrm{IRR}>4 \%$ & Scenario 1 & Bituminous low volatiles & $23,117,328$ & 8 & 4.5 & 5 \\
\hline IRR $>4 \%$ & Scenario 1 & Bituminous low volatiles & $14,384,115$ & 8 & 2.8 & 7.5 \\
\hline $\mathrm{IRR}>4 \%$ & Scenario 1 & Bituminous low volatiles & $11,815,523$ & 8 & 2.3 & 10 \\
\hline $\mathrm{IRR}>4 \%$ & Scenario 1 & Bituminous medium volatiles & $224,751,800$ & 7 & 50 & 1 \\
\hline $\mathrm{IRR}>4 \%$ & Scenario 1 & Bituminous medium volatiles & $47,197,878$ & 7 & 10.5 & 2.5 \\
\hline $\mathrm{IRR}>4 \%$ & Scenario 1 & Bituminous medium volatiles & $20,227,662$ & 7 & 4.5 & 5 \\
\hline $\mathrm{IRR}>4 \%$ & Scenario 1 & Bituminous medium volatiles & $12,586,101$ & 7 & 2.8 & 7.5 \\
\hline $\mathrm{IRR}>4 \%$ & Scenario 1 & Bituminous medium volatiles & $10,338,583$ & 7 & 2.3 & 10 \\
\hline $\mathrm{IRR}>4 \%$ & Scenario 1 & Bituminous high volatiles A & $192,644,400$ & 6 & 50 & 1 \\
\hline $\mathrm{IRR}>4 \%$ & Scenario 1 & Bituminous high volatiles A & $40,455,324$ & 6 & 10.5 & 2.5 \\
\hline $\mathrm{IRR}>4 \%$ & Scenario 1 & Bituminous high volatiles A & $17,337,996$ & 6 & 4.5 & 5 \\
\hline $\mathrm{IRR}>4 \%$ & Scenario 1 & Bituminous high volatiles A & $10,788,086$ & 6 & 2.8 & 7.5 \\
\hline $\mathrm{IRR}>4 \%$ & Scenario 1 & Bituminous high volatiles A & $8,861,642$ & 6 & 2.3 & 10 \\
\hline $\mathrm{IRR}>4 \%$ & Scenario 1 & Bituminous high volatiles B & $160,537,000$ & 5 & 50 & 1 \\
\hline $\mathrm{IRR}>4 \%$ & Scenario 1 & Bituminous high volatiles B & $33,712,770$ & 5 & 10.5 & 2.5 \\
\hline $\mathrm{IRR}>4 \%$ & Scenario 1 & Bituminous high volatiles B & $14,448,330$ & 5 & 4.5 & 5 \\
\hline $\mathrm{IRR}>4 \%$ & Scenario 1 & Bituminous high volatiles B & $8,990,072$ & 5 & 2.8 & 7.5 \\
\hline IRR $>4 \%$ & Scenario 1 & Bituminous high volatiles B & $7,384,702$ & 5 & 2.3 & 10 \\
\hline IRR & Price & Rank & GIP area min & GIS min. & Height min. & Perm \\
\hline 30 years & $€ / \mathrm{m}^{3}$ & & $\mathrm{~m}^{3}$ & $\mathrm{~m}^{3} / \mathrm{tp}$ & $\mathrm{m}$ & $\mathrm{mD}$ \\
\hline IRR $>4 \%$ & Scenario 2 & Anthracite & $462,346,560$ & 12 & 60 & 1 \\
\hline $\mathrm{IRR}>4 \%$ & Scenario 2 & Anthracite & $100,175,088$ & 12 & 13.0 & 2.5 \\
\hline $\mathrm{IRR}>4 \%$ & Scenario 2 & Anthracite & $42,381,768$ & 12 & 5.5 & 5 \\
\hline $\mathrm{IRR}>4 \%$ & Scenario 2 & Anthracite & $26,970,216$ & 12 & 3.5 & 7.5 \\
\hline $\mathrm{IRR}>4 \%$ & Scenario 2 & Anthracite & $22,346,750$ & 12 & 2.9 & 10 \\
\hline $\mathrm{IRR}>4 \%$ & Scenario 2 & Bituminous low volatiles & $308,231,040$ & 8 & 60 & 1 \\
\hline $\mathrm{IRR}>4 \%$ & Scenario 2 & Bituminous low volatiles & $66,783,392$ & 8 & 13.0 & 2.5 \\
\hline $\mathrm{IRR}>4 \%$ & Scenario 2 & Bituminous low volatiles & $28,254,512$ & 8 & 5.5 & 5 \\
\hline $\mathrm{IRR}>4 \%$ & Scenario 2 & Bituminous low volatiles & $17,980,144$ & 8 & 3.5 & 7.5 \\
\hline IRR $>4 \%$ & Scenario 2 & Bituminous low volatiles & $14,897,834$ & 8 & 2.9 & 10 \\
\hline IRR $>4 \%$ & Scenario 2 & Bituminous medium volatiles & $269,702,160$ & 7 & 60 & 1 \\
\hline
\end{tabular}




\section{Continued}

\begin{tabular}{|c|c|c|c|c|c|c|}
\hline IRR $>4 \%$ & Scenario 2 & Bituminous medium volatiles & $58,435,468$ & 7 & 13.0 & 2.5 \\
\hline $\mathrm{IRR}>4 \%$ & Scenario 2 & Bituminous medium volatiles & $24,722,698$ & 7 & 5.5 & 5 \\
\hline $\mathrm{IRR}>4 \%$ & Scenario 2 & Bituminous medium volatiles & $15,732,626$ & 7 & 3.5 & 7.5 \\
\hline IRR $>4 \%$ & Scenario 2 & Bituminous medium volatiles & $13,035,604$ & 7 & 2.9 & 10 \\
\hline $\mathrm{IRR}>4 \%$ & Scenario 2 & Bituminous high volatiles A & $231,173,280$ & 6 & 60 & 1 \\
\hline $\mathrm{IRR}>4 \%$ & Scenario 2 & Bituminous high volatiles A & $50,087,544$ & 6 & 13.0 & 2.5 \\
\hline IRR $>4 \%$ & Scenario 2 & Bituminous high volatiles A & $21,190,884$ & 6 & 5.5 & 5 \\
\hline IRR $>4 \%$ & Scenario 2 & Bituminous high volatiles A & $13,485,108$ & 6 & 3.5 & 7.5 \\
\hline $\mathrm{IRR}>4 \%$ & Scenario 2 & Bituminous high volatiles A & $11,173,375$ & 6 & 2.9 & 10 \\
\hline $\mathrm{IRR}>4 \%$ & Scenario 2 & Bituminous high volatiles B & $192,644,400$ & 5 & 60 & 1 \\
\hline IRR $>4 \%$ & Scenario 2 & Bituminous high volatiles B & $41,739,620$ & 5 & 13.0 & 2.5 \\
\hline $\mathrm{IRR}>4 \%$ & Scenario 2 & Bituminous high volatiles B & $17,659,070$ & 5 & 5.5 & 5 \\
\hline IRR $>4 \%$ & Scenario 2 & Bituminous high volatiles B & $11,237,590$ & 5 & 3.5 & 7.5 \\
\hline $\mathrm{IRR}>4 \%$ & Scenario 2 & Bituminous high volatiles B & $9,311,146$ & 5 & 2.9 & 10 \\
\hline IRR & Price & Rank & GIP area min & GIS min. & Height min. & Perm \\
\hline 30 years & $€ / \mathrm{m}^{3}$ & & $\mathrm{~m}^{3}$ & $\mathrm{~m}^{3} / \mathrm{tp}$ & $\mathrm{m}$ & $\mathrm{mD}$ \\
\hline $\mathrm{IRR}>4 \%$ & Scenario 3 & Anthracite & $308,231,040$ & 12 & 40 & 1 \\
\hline $\mathrm{IRR}>4 \%$ & Scenario 3 & Anthracite & $65,499,096$ & 12 & 8.5 & 2.5 \\
\hline $\mathrm{IRR}>4 \%$ & Scenario 3 & Anthracite & $25,429,061$ & 12 & 3.3 & 5 \\
\hline IRR $>4 \%$ & Scenario 3 & Anthracite & $18,493,862$ & 12 & 2.4 & 7.5 \\
\hline $\mathrm{IRR}>4 \%$ & Scenario 3 & Anthracite & $13,870,397$ & 12 & 1.8 & 10 \\
\hline IRR $>4 \%$ & Scenario 3 & Bituminous low volatiles & $205,487,360$ & 8 & 40 & 1 \\
\hline $\mathrm{IRR}>4 \%$ & Scenario 3 & Bituminous low volatiles & $43,666,064$ & 8 & 8.5 & 2.5 \\
\hline IRR $>4 \%$ & Scenario 3 & Bituminous low volatiles & $16,952,707$ & 8 & 3.3 & 5 \\
\hline $\mathrm{IRR}>4 \%$ & Scenario 3 & Bituminous low volatiles & $12,329,242$ & 8 & 2.4 & 7.5 \\
\hline $\mathrm{IRR}>4 \%$ & Scenario 3 & Bituminous low volatiles & $9,246,931$ & 8 & 1.8 & 10 \\
\hline $\mathrm{IRR}>4 \%$ & Scenario 3 & Bituminous medium volatiles & $179,801,440$ & 7 & 40 & 1 \\
\hline $\mathrm{IRR}>4 \%$ & Scenario 3 & Bituminous medium volatiles & $38,207,806$ & 7 & 8.5 & 2.5 \\
\hline $\mathrm{IRR}>4 \%$ & Scenario 3 & Bituminous medium volatiles & $14,833,619$ & 7 & 3.3 & 5 \\
\hline $\mathrm{IRR}>4 \%$ & Scenario 3 & Bituminous medium volatiles & $10,788,086$ & 7 & 2.4 & 7.5 \\
\hline $\mathrm{IRR}>4 \%$ & Scenario 3 & Bituminous medium volatiles & $8,091,065$ & 7 & 1.8 & 10 \\
\hline $\mathrm{IRR}>4 \%$ & Scenario 3 & Bituminous high volatiles A & $154,115,520$ & 6 & 40 & 1 \\
\hline IRR $>4 \%$ & Scenario 3 & Bituminous high volatiles A & $32,749,548$ & 6 & 8.5 & 2.5 \\
\hline $\mathrm{IRR}>4 \%$ & Scenario 3 & Bituminous high volatiles A & $12,714,530$ & 6 & 3.3 & 5 \\
\hline $\mathrm{IRR}>4 \%$ & Scenario 3 & Bituminous high volatiles A & $9,246,931$ & 6 & 2.4 & 7.5 \\
\hline $\mathrm{IRR}>4 \%$ & Scenario 3 & Bituminous high volatiles A & $6,935,198$ & 6 & 1.8 & 10 \\
\hline IRR $>4 \%$ & Scenario 3 & Bituminous high volatiles B & $128,429,600$ & 5 & 40 & 1 \\
\hline
\end{tabular}




\section{Continued}

\begin{tabular}{|c|c|c|c|c|c|c|}
\hline IRR $>4 \%$ & Scenario 3 & Bituminous high volatiles $B$ & $27,291,290$ & 5 & 8.5 & 2.5 \\
\hline IRR $>4 \%$ & Scenario 3 & Bituminous high volatiles B & $10,595,442$ & 5 & 3.3 & 5 \\
\hline IRR $>4 \%$ & Scenario 3 & Bituminous high volatiles B & $7,705,776$ & 5 & 2.4 & 7.5 \\
\hline IRR $>4 \%$ & Scenario 3 & Bituminous high volatiles B & $5,779,332$ & 5 & 1.8 & 10 \\
\hline IRR & Price & Rank & GIP area min & GIS min. & Height min. & Perm \\
\hline 30 years & $€ / \mathrm{m}^{3}$ & & $\mathrm{~m}^{3}$ & $\mathrm{~m}^{3} / \mathrm{tp}$ & $\mathrm{m}$ & $\mathrm{mD}$ \\
\hline IRR $>4 \%$ & Scenario 4 & Anthracite & $269,702,160$ & 12 & 35 & 1 \\
\hline IRR $>4 \%$ & Scenario 4 & Anthracite & $53,940,432$ & 12 & 7.0 & 2.5 \\
\hline IRR $>4 \%$ & Scenario 4 & Anthracite & $20,805,595$ & 12 & 2.7 & 5 \\
\hline IRR $>4 \%$ & Scenario 4 & Anthracite & $15,411,552$ & 12 & 2.0 & 7.5 \\
\hline IRR $>4 \%$ & Scenario 4 & Anthracite & $11,558,664$ & 12 & 1.5 & 10 \\
\hline IRR $>4 \%$ & Scenario 4 & Bituminous low volatiles & $179,801,440$ & 8 & 35 & 1 \\
\hline IRR $>4 \%$ & Scenario 4 & Bituminous low volatiles & $35,960,288$ & 8 & 7.0 & 2.5 \\
\hline IRR $>4 \%$ & Scenario 4 & Bituminous low volatiles & $13,870,397$ & 8 & 2.7 & 5 \\
\hline IRR $>4 \%$ & Scenario 4 & Bituminous low volatiles & $10,274,368$ & 8 & 2.0 & 7.5 \\
\hline IRR $>4 \%$ & Scenario 4 & Bituminous low volatiles & $7,705,776$ & 8 & 1.5 & 10 \\
\hline IRR $>4 \%$ & Scenario 4 & Bituminous medium volatiles & $157,326,260$ & 7 & 35 & 1 \\
\hline IRR $>4 \%$ & Scenario 4 & Bituminous medium volatiles & $31,465,252$ & 7 & 7.0 & 2.5 \\
\hline IRR $>4 \%$ & Scenario 4 & Bituminous medium volatiles & $12,136,597$ & 7 & 2.7 & 5 \\
\hline IRR $>4 \%$ & Scenario 4 & Bituminous medium volatiles & $8,990,072$ & 7 & 2.0 & 7.5 \\
\hline IRR $>4 \%$ & Scenario 4 & Bituminous medium volatiles & $6,742,554$ & 7 & 1.5 & 10 \\
\hline IRR $>4 \%$ & Scenario 4 & Bituminous high volatiles A & $134,851,080$ & 6 & 35 & 1 \\
\hline IRR $>4 \%$ & Scenario 4 & Bituminous high volatiles A & $26,970,216$ & 6 & 7.0 & 2.5 \\
\hline IRR $>4 \%$ & Scenario 4 & Bituminous high volatiles A & $10,402,798$ & 6 & 2.7 & 5 \\
\hline IRR $>4 \%$ & Scenario 4 & Bituminous high volatiles A & $7,705,776$ & 6 & 2.0 & 7.5 \\
\hline IRR $>4 \%$ & Scenario 4 & Bituminous high volatiles A & $5,779,332$ & 6 & 1.5 & 10 \\
\hline IRR $>4 \%$ & Scenario 4 & Bituminous high volatiles B & $112,375,900$ & 5 & 35 & 1 \\
\hline $\mathrm{IRR}>4 \%$ & Scenario 4 & Bituminous high volatiles B & $22,475,180$ & 5 & 7.0 & 2.5 \\
\hline IRR $>4 \%$ & Scenario 4 & Bituminous high volatiles B & $8,668,998$ & 5 & 2.7 & 5 \\
\hline IRR $>4 \%$ & Scenario 4 & Bituminous high volatiles B & $6,421,480$ & 5 & 2.0 & 7,5 \\
\hline IRR $>4 \%$ & Scenario 4 & Bituminous high volatiles B & $4,816,110$ & 5 & 1.5 & 10 \\
\hline IRR & Price & Rank & GIP area min & GIS min. & Height min, & Perm \\
\hline 30 years & $€ / \mathrm{m}^{3}$ & & $\mathrm{~m}^{3}$ & $\mathrm{~m}^{3} / \mathrm{tp}$ & $\mathrm{m}$ & $\mathrm{mD}$ \\
\hline IRR $>8 \%$ & Scenario 1 & Anthracite & $408,406,128$ & 12 & 53 & 1 \\
\hline IRR $>8 \%$ & Scenario 1 & Anthracite & $92,469,312$ & 12 & 12 & 2.5 \\
\hline IRR $>8 \%$ & Scenario 1 & Anthracite & $35,446,570$ & 12 & 4,6 & 5 \\
\hline IRR $>8 \%$ & Scenario 1 & Anthracite & $23,887,906$ & 12 & 3.1 & 7.5 \\
\hline
\end{tabular}




\section{Continued}

\begin{tabular}{|c|c|c|c|c|c|c|}
\hline IRR $>8 \%$ & Scenario 1 & Anthracite & $19,264,440$ & 12 & 2.5 & 10 \\
\hline IRR $>8 \%$ & Scenario 1 & Bituminous low volatiles & $272,270,752$ & 8 & 53 & 1 \\
\hline IRR $>8 \%$ & Scenario 1 & Bituminous low volatiles & $61,646,208$ & 8 & 12 & 2,5 \\
\hline IRR $>8 \%$ & Scenario 1 & Bituminous low volatiles & $23,631,046$ & 8 & 4.6 & 5 \\
\hline IRR $>8 \%$ & Scenario 1 & Bituminous low volatiles & $15,925,270$ & 8 & 3.1 & 7.5 \\
\hline IRR $>8 \%$ & Scenario 1 & Bituminous low volatiles & $12,842,960$ & 8 & 2.5 & 10 \\
\hline IRR $>8 \%$ & Scenario 1 & Bituminous medium volatiles & $238,236,908$ & 7 & 53 & 1 \\
\hline IRR $>8 \%$ & Scenario 1 & Bituminous medium volatiles & $53,940,432$ & 7 & 12 & 2.5 \\
\hline IRR $>8 \%$ & Scenario 1 & Bituminous medium volatiles & $20,677,166$ & 7 & 4.6 & 5 \\
\hline $\mathrm{IRR}>8 \%$ & Scenario 1 & Bituminous medium volatiles & $13,934,612$ & 7 & 3.1 & 7.5 \\
\hline IRR $>8 \%$ & Scenario 1 & Bituminous medium volatiles & $11,237,590$ & 7 & 2.5 & 10 \\
\hline $\mathrm{IRR}>8 \%$ & Scenario 1 & Bituminous high volatiles A & $204,203,064$ & 6 & 53 & 1 \\
\hline IRR $>8 \%$ & Scenario 1 & Bituminous high volatiles A & $46,234,656$ & 6 & 12 & 2.5 \\
\hline $\mathrm{IRR}>8 \%$ & Scenario 1 & Bituminous high volatiles A & $17,723,285$ & 6 & 4.6 & 5 \\
\hline IRR $>8 \%$ & Scenario 1 & Bituminous high volatiles A & $11,943,953$ & 6 & 3.1 & 7.5 \\
\hline $\mathrm{IRR}>8 \%$ & Scenario 1 & Bituminous high volatiles A & $9,632,220$ & 6 & 2.5 & 10 \\
\hline IRR $>8 \%$ & Scenario 1 & Bituminous high volatiles B & $170,169,220$ & 5 & 53 & 1 \\
\hline IRR $>8 \%$ & Scenario 1 & Bituminous high volatiles B & $38,528,880$ & 5 & 12 & 2.5 \\
\hline $\mathrm{IRR}>8 \%$ & Scenario 1 & Bituminous high volatiles B & $14,769,404$ & 5 & 4.6 & 5 \\
\hline IRR $>8 \%$ & Scenario 1 & Bituminous high volatiles B & $9,953,294$ & 5 & 3.1 & 7.5 \\
\hline IRR $>8 \%$ & Scenario 1 & Bituminous high volatiles B & $8,026,850$ & 5 & 2.5 & 10 \\
\hline IRR & Price & Rank & GIP area min & GIS min. & Height min. & Perm \\
\hline 30 years & $€ / \mathrm{m}^{3}$ & & $\mathrm{~m}^{3}$ & $\mathrm{~m}^{3} / \mathrm{tp}$ & $\mathrm{m}$ & $\mathrm{mD}$ \\
\hline IRR $>8 \%$ & Scenario 2 & Anthracite & $508,581,216$ & 12 & 66 & 1 \\
\hline IRR $>8 \%$ & Scenario 2 & Anthracite & $100,175,088$ & 12 & 13 & 2.5 \\
\hline IRR $>8 \%$ & Scenario 2 & Anthracite & $43,922,923$ & 12 & 5.7 & 5 \\
\hline IRR $>8 \%$ & Scenario 2 & Anthracite & $26,970,216$ & 12 & 3.5 & 7.5 \\
\hline IRR $>8 \%$ & Scenario 2 & Anthracite & $23,887,906$ & 12 & 3.1 & 10 \\
\hline IRR $>8 \%$ & Scenario 2 & Bituminous low volatiles & $339,054,144$ & 8 & 66 & 1 \\
\hline IRR $>8 \%$ & Scenario 2 & Bituminous low volatiles & $66,783,392$ & 8 & 13 & 2.5 \\
\hline $\mathrm{IRR}>8 \%$ & Scenario 2 & Bituminous low volatiles & $29,281,949$ & 8 & 5.7 & 5 \\
\hline IRR $>8 \%$ & Scenario 2 & Bituminous low volatiles & $17,980,144$ & 8 & 3.5 & 7.5 \\
\hline $\mathrm{IRR}>8 \%$ & Scenario 2 & Bituminous low volatiles & $15,925,270$ & 8 & 3.1 & 10 \\
\hline IRR $>8 \%$ & Scenario 2 & Bituminous medium volatiles & $296,672,376$ & 7 & 66 & 1 \\
\hline IRR $>8 \%$ & Scenario 2 & Bituminous medium volatiles & $58,435,468$ & 7 & 13 & 2.5 \\
\hline IRR $>8 \%$ & Scenario 2 & Bituminous medium volatiles & $25,621,705$ & 7 & 5.7 & 5 \\
\hline IRR $>8 \%$ & Scenario 2 & Bituminous medium volatiles & $15,732,626$ & 7 & 3.5 & 7.5 \\
\hline
\end{tabular}




\section{Continued}

\begin{tabular}{|c|c|c|c|c|c|c|}
\hline IRR $>8 \%$ & Scenario 2 & Bituminous medium volatiles & $13,934,612$ & 7 & 3.1 & 10 \\
\hline IRR $>8 \%$ & Scenario 2 & Bituminous high volatiles A & $254,290,608$ & 6 & 66 & 1 \\
\hline $\mathrm{IRR}>8 \%$ & Scenario 2 & Bituminous high volatiles A & $50,087,544$ & 6 & 13 & 2.5 \\
\hline IRR $>8 \%$ & Scenario 2 & Bituminous high volatiles A & $21,961,462$ & 6 & 5.7 & 5 \\
\hline $\mathrm{IRR}>8 \%$ & Scenario 2 & Bituminous high volatiles A & $13,485,108$ & 6 & 3.5 & 7.5 \\
\hline IRR $>8 \%$ & Scenario 2 & Bituminous high volatiles A & $11,943,953$ & 6 & 3.1 & 10 \\
\hline IRR $>8 \%$ & Scenario 2 & Bituminous high volatiles B & $211,908,840$ & 5 & 66 & 1 \\
\hline IRR $>8 \%$ & Scenario 2 & Bituminous high volatiles B & $41,739,620$ & 5 & 13 & 2.5 \\
\hline IRR $>8 \%$ & Scenario 2 & Bituminous high volatiles B & $18,301,218$ & 5 & 5.7 & 5 \\
\hline IRR $>8 \%$ & Scenario 2 & Bituminous high volatiles $B$ & $11,237,590$ & 5 & 3.5 & 7.5 \\
\hline IRR $>8 \%$ & Scenario 2 & Bituminous high volatiles B & $9,953,294$ & 5 & 3.1 & 10 \\
\hline IRR & Price & Rank & GIP area min & GIS min. & Height min. & Perm \\
\hline 30 years & $€ / \mathrm{m}^{3}$ & & $\mathrm{~m}^{3}$ & $\mathrm{~m}^{3} / \mathrm{tp}$ & $\mathrm{m}$ & $\mathrm{mD}$ \\
\hline IRR $>8 \%$ & Scenario 3 & Anthracite & $323,642,592$ & 12 & 42 & 1 \\
\hline IRR $>8 \%$ & Scenario 3 & Anthracite & $69,351,984$ & 12 & 9 & 2.5 \\
\hline IRR $>8 \%$ & Scenario 3 & Anthracite & $27,740,794$ & 12 & 3.6 & 5 \\
\hline IRR $>8 \%$ & Scenario 3 & Anthracite & $19,264,440$ & 12 & 2.5 & 7.5 \\
\hline $\mathrm{IRR}>8 \%$ & Scenario 3 & Anthracite & $15,411,552$ & 12 & 2.0 & 10 \\
\hline $\mathrm{IRR}>8 \%$ & Scenario 3 & Bituminous low volatiles & $215,761,728$ & 8 & 42 & 1 \\
\hline IRR $>8 \%$ & Scenario 3 & Bituminous low volatiles & $46,234,656$ & 8 & 9 & 2.5 \\
\hline $\mathrm{IRR}>8 \%$ & Scenario 3 & Bituminous low volatiles & $18,493,862$ & 8 & 3.6 & 5 \\
\hline $\mathrm{IRR}>8 \%$ & Scenario 3 & Bituminous low volatiles & $12,842,960$ & 8 & 2.5 & 7.5 \\
\hline $\mathrm{IRR}>8 \%$ & Scenario 3 & Bituminous low volatiles & $10,274,368$ & 8 & 2.0 & 10 \\
\hline IRR $>8 \%$ & Scenario 3 & Bituminous medium volatiles & $188,791,512$ & 7 & 42 & 1 \\
\hline IRR $>8 \%$ & Scenario 3 & Bituminous medium volatiles & $40,455,324$ & 7 & 9 & 2.5 \\
\hline IRR $>8 \%$ & Scenario 3 & Bituminous medium volatiles & $16,182,130$ & 7 & 3.6 & 5 \\
\hline $\mathrm{IRR}>8 \%$ & Scenario 3 & Bituminous medium volatiles & $11,237,590$ & 7 & 2.5 & 7.5 \\
\hline $\mathrm{IRR}>8 \%$ & Scenario 3 & Bituminous medium volatiles & $8,990,072$ & 7 & 2.0 & 10 \\
\hline $\mathrm{IRR}>8 \%$ & Scenario 3 & Bituminous high volatiles A & $161,821,296$ & 6 & 42 & 1 \\
\hline IRR $>8 \%$ & Scenario 3 & Bituminous high volatiles A & $34,675,992$ & 6 & 9 & 2.5 \\
\hline $\mathrm{IRR}>8 \%$ & Scenario 3 & Bituminous high volatiles A & $13,870,397$ & 6 & 3.6 & 5 \\
\hline $\mathrm{IRR}>8 \%$ & Scenario 3 & Bituminous high volatiles A & $9,632,220$ & 6 & 2.5 & 7.5 \\
\hline IRR $>8 \%$ & Scenario 3 & Bituminous high volatiles A & $7,705,776$ & 6 & 2.0 & 10 \\
\hline $\mathrm{IRR}>8 \%$ & Scenario 3 & Bituminous high volatiles B & $134,851,080$ & 5 & 42 & 1 \\
\hline IRR $>8 \%$ & Scenario 3 & Bituminous high volatiles B & $28,896,660$ & 5 & 9 & 2.5 \\
\hline $\mathrm{IRR}>8 \%$ & Scenario 3 & Bituminous high volatiles $B$ & $11,558,664$ & 5 & 3.6 & 5 \\
\hline IRR $>8 \%$ & Scenario 3 & Bituminous high volatiles B & $8,026,850$ & 5 & 2.5 & 7.5 \\
\hline
\end{tabular}




\section{Continued}

\begin{tabular}{|c|c|c|c|c|c|c|}
\hline IRR $>8 \%$ & Scenario 3 & Bituminous high volatiles $B$ & $6,421,480$ & 5 & 2.0 & 10 \\
\hline IRR & Price & Rank & GIP area min & GIS min. & Height min. & Perm \\
\hline 30 years & $€ / \mathrm{m}^{3}$ & & $\mathrm{~m}^{3}$ & $\mathrm{~m}^{3} / \mathrm{tp}$ & $\mathrm{m}$ & $\mathrm{mD}$ \\
\hline IRR $>8 \%$ & Scenario 4 & Anthracite & $269,702,160$ & 12 & 35 & 1 \\
\hline IRR $>8 \%$ & Scenario 4 & Anthracite & $57,793,320$ & 12 & 7.5 & 2.5 \\
\hline IRR $>8 \%$ & Scenario 4 & Anthracite & $23,117,328$ & 12 & 3 & 5 \\
\hline IRR $>8 \%$ & Scenario 4 & Anthracite & $16,182,130$ & 12 & 2.1 & 7.5 \\
\hline IRR $>8 \%$ & Scenario 4 & Anthracite & $13,099,819$ & 12 & 1.7 & 10 \\
\hline IRR $>8 \%$ & Scenario 4 & Bituminous low volatiles & $179,801,440$ & 8 & 35 & 1 \\
\hline IRR $>8 \%$ & Scenario 4 & Bituminous low volatiles & $38,528,880$ & 8 & 7.5 & 2.5 \\
\hline IRR $>8 \%$ & Scenario 4 & Bituminous low volatiles & $15,411,552$ & 8 & 3 & 5 \\
\hline IRR $>8 \%$ & Scenario 4 & Bituminous low volatiles & $10,788,086$ & 8 & 2.1 & 7.5 \\
\hline IRR $>8 \%$ & Scenario 4 & Bituminous low volatiles & $8,733,213$ & 8 & 1.7 & 10 \\
\hline IRR $>8 \%$ & Scenario 4 & Bituminous medium volatiles & $157,326,260$ & 7 & 35 & 1 \\
\hline IRR $>8 \%$ & Scenario 4 & Bituminous medium volatiles & $33,712,770$ & 7 & 7.5 & 2.5 \\
\hline IRR $>8 \%$ & Scenario 4 & Bituminous medium volatiles & $13,485,108$ & 7 & 3 & 5 \\
\hline IRR $>8 \%$ & Scenario 4 & Bituminous medium volatiles & $9,439,576$ & 7 & 2.1 & 7.5 \\
\hline IRR $>8 \%$ & Scenario 4 & Bituminous medium volatiles & $7,641,561$ & 7 & 1.7 & 10 \\
\hline IRR $>8 \%$ & Scenario 4 & Bituminous high volatiles A & $134,851,080$ & 6 & 35 & 1 \\
\hline IRR $>8 \%$ & Scenario 4 & Bituminous high volatiles A & $28,896,660$ & 6 & 7.5 & 2.5 \\
\hline IRR $>8 \%$ & Scenario 4 & Bituminous high volatiles A & $11,558,664$ & 6 & 3 & 5 \\
\hline IRR $>8 \%$ & Scenario 4 & Bituminous high volatiles A & $8,091,065$ & 6 & 2.1 & 7.5 \\
\hline IRR $>8 \%$ & Scenario 4 & Bituminous high volatiles A & $6,549,910$ & 6 & 1.7 & 10 \\
\hline IRR $>8 \%$ & Scenario 4 & Bituminous high volatiles $B$ & $112,375,900$ & 5 & 35 & 1 \\
\hline IRR $>8 \%$ & Scenario 4 & Bituminous high volatiles B & $24,080,550$ & 5 & 7.5 & 2.5 \\
\hline IRR $>8 \%$ & Scenario 4 & Bituminous high volatiles B & $9,632,220$ & 5 & 3 & 5 \\
\hline IRR $>8 \%$ & Scenario 4 & Bituminous high volatiles $B$ & $6,742,554$ & 5 & 2.1 & 7.5 \\
\hline IRR $>8 \%$ & Scenario 4 & Bituminous high volatiles $B$ & $5,458,258$ & 5 & 1.7 & 10 \\
\hline IRR & Price & Rank & GIP area min & GIS min. & Height min. & Perm \\
\hline 10 years & $€ / \mathrm{m}^{3}$ & & $\mathrm{~m}^{3}$ & $\mathrm{~m}^{3} / \mathrm{tp}$ & $\mathrm{m}$ & $\mathrm{mD}$ \\
\hline IRR $>0 \%$ & Scenario 1 & Anthracite & $755,166,048$ & 12 & 98 & 1 \\
\hline IRR $>0 \%$ & Scenario 1 & Anthracite & $161,821,296$ & 12 & 21.0 & 2.5 \\
\hline IRR $>0 \%$ & Scenario 1 & Anthracite & $45,464,078$ & 12 & 5.9 & 5 \\
\hline IRR $>0 \%$ & Scenario 1 & Anthracite & $24,658,483$ & 12 & 3.2 & 7.5 \\
\hline IRR $>0 \%$ & Scenario 1 & Anthracite & $16,182,130$ & 12 & 2.10 & 10 \\
\hline IRR $>0 \%$ & Scenario 1 & Bituminous low volatiles & $503,444,032$ & 8 & 98 & 1 \\
\hline IRR $>0 \%$ & Scenario 1 & Bituminous low volatiles & $107,880,864$ & 8 & 21.0 & 2.5 \\
\hline
\end{tabular}




\section{Continued}

\begin{tabular}{|c|c|c|c|c|c|c|}
\hline $\mathrm{IRR}>0 \%$ & Scenario 1 & Bituminous low volatiles & $30,309,386$ & 8 & 5.9 & 5 \\
\hline IRR $>0 \%$ & Scenario 1 & Bituminous low volatiles & $16,438,989$ & 8 & 3.2 & 7.5 \\
\hline $\mathrm{IRR}>0 \%$ & Scenario 1 & Bituminous low volatiles & $10,788,086$ & 8 & 2.10 & 10 \\
\hline $\mathrm{IRR}>0 \%$ & Scenario 1 & Bituminous medium volatiles & $440,513,528$ & 7 & 98 & 1 \\
\hline $\mathrm{IRR}>0 \%$ & Scenario 1 & Bituminous medium volatiles & $94,395,756$ & 7 & 21.0 & 2.5 \\
\hline IRR $>0 \%$ & Scenario 1 & Bituminous medium volatiles & $26,520,712$ & 7 & 5.9 & 5 \\
\hline IRR $>0 \%$ & Scenario 1 & Bituminous medium volatiles & $14,384,115$ & 7 & 3.2 & 7.5 \\
\hline $\mathrm{IRR}>0 \%$ & Scenario 1 & Bituminous medium volatiles & $9,439,576$ & 7 & 2.10 & 10 \\
\hline $\mathrm{IRR}>0 \%$ & Scenario 1 & Bituminous high volatiles A & $377,583,024$ & 6 & 98 & 1 \\
\hline $\mathrm{IRR}>0 \%$ & Scenario 1 & Bituminous high volatiles A & $80,910,648$ & 6 & 21.0 & 2.5 \\
\hline $\mathrm{IRR}>0 \%$ & Scenario 1 & Bituminous high volatiles A & $22,732,039$ & 6 & 5.9 & 5 \\
\hline $\mathrm{IRR}>0 \%$ & Scenario 1 & Bituminous high volatiles A & $12,329,242$ & 6 & 3.2 & 7.5 \\
\hline IRR $>0 \%$ & Scenario 1 & Bituminous high volatiles A & $8,091,065$ & 6 & 2.10 & 10 \\
\hline $\mathrm{IRR}>0 \%$ & Scenario 1 & Bituminous high volatiles B & $314,652,520$ & 5 & 98 & 1 \\
\hline $\mathrm{IRR}>0 \%$ & Scenario 1 & Bituminous high volatiles B & $67,425,540$ & 5 & 21.0 & 2.5 \\
\hline IRR $>0 \%$ & Scenario 1 & Bituminous high volatiles $B$ & $18,943,366$ & 5 & 5.9 & 5 \\
\hline $\mathrm{IRR}>0 \%$ & Scenario 1 & Bituminous high volatiles B & $10,274,368$ & 5 & 3.2 & 7.5 \\
\hline $\mathrm{IRR}>0 \%$ & Scenario 1 & Bituminous high volatiles $B$ & $6,742,554$ & 5 & 2.10 & 10 \\
\hline IRR & Price & Rank & GIP area min & GIS min. & Height min. & Perm \\
\hline 10 years & $€ / \mathrm{m}^{3}$ & & $\mathrm{~m}^{3}$ & $\mathrm{~m}^{3} / \mathrm{tp}$ & $\mathrm{m}$ & $\mathrm{mD}$ \\
\hline IRR $>0 \%$ & Scenario 2 & Anthracite & $1,001,750,880$ & 12 & 130 & 1 \\
\hline $\mathrm{IRR}>0 \%$ & Scenario 2 & Anthracite & $200,350,176$ & 12 & 26.0 & 2.5 \\
\hline $\mathrm{IRR}>0 \%$ & Scenario 2 & Anthracite & $57,793,320$ & 12 & 7.5 & 5 \\
\hline IRR $>0 \%$ & Scenario 2 & Anthracite & $30,823,104$ & 12 & 4.0 & 7.5 \\
\hline $\mathrm{IRR}>0 \%$ & Scenario 2 & Anthracite & $20,189,133$ & 12 & 2.62 & 10 \\
\hline IRR $>0 \%$ & Scenario 2 & Bituminous low volatiles & $667,833,920$ & 8 & 130 & 1 \\
\hline $\mathrm{IRR}>0 \%$ & Scenario 2 & Bituminous low volatiles & $133,566,784$ & 8 & 26.0 & 2.5 \\
\hline $\mathrm{IRR}>0 \%$ & Scenario 2 & Bituminous low volatiles & $38,528,880$ & 8 & 7.5 & 5 \\
\hline $\mathrm{IRR}>0 \%$ & Scenario 2 & Bituminous low volatiles & $20,548,736$ & 8 & 4.0 & 7.5 \\
\hline IRR $>0 \%$ & Scenario 2 & Bituminous low volatiles & $13,459,422$ & 8 & 2.62 & 10 \\
\hline $\mathrm{IRR}>0 \%$ & Scenario 2 & Bituminous medium volatiles & $584,354,680$ & 7 & 130 & 1 \\
\hline IRR $>0 \%$ & Scenario 2 & Bituminous medium volatiles & $116,870,936$ & 7 & 26.0 & 2.5 \\
\hline IRR $>0 \%$ & Scenario 2 & Bituminous medium volatiles & $33,712,770$ & 7 & 7.5 & 5 \\
\hline $\mathrm{IRR}>0 \%$ & Scenario 2 & Bituminous medium volatiles & $17,980,144$ & 7 & 4.0 & 7.5 \\
\hline IRR $>0 \%$ & Scenario 2 & Bituminous medium volatiles & $11,776,994$ & 7 & 2.62 & 10 \\
\hline $\mathrm{IRR}>0 \%$ & Scenario 2 & Bituminous high volatiles A & $500,875,440$ & 6 & 130 & 1 \\
\hline IRR $>0 \%$ & Scenario 2 & Bituminous high volatiles A & $100,175,088$ & 6 & 26.0 & 2.5 \\
\hline
\end{tabular}




\section{Continued}

\begin{tabular}{|c|c|c|c|c|c|c|}
\hline IRR $>0 \%$ & Scenario 2 & Bituminous high volatiles A & $28,896,660$ & 6 & 7.5 & 5 \\
\hline IRR $>0 \%$ & Scenario 2 & Bituminous high volatiles A & $15,411,552$ & 6 & 4.0 & 7.5 \\
\hline IRR $>0 \%$ & Scenario 2 & Bituminous high volatiles A & $10,094,567$ & 6 & 2.62 & 10 \\
\hline IRR $>0 \%$ & Scenario 2 & Bituminous high volatiles B & $417,396,200$ & 5 & 130 & 1 \\
\hline IRR $>0 \%$ & Scenario 2 & Bituminous high volatiles B & $83,479,240$ & 5 & 26.0 & 2.5 \\
\hline IRR $>0 \%$ & Scenario 2 & Bituminous high volatiles B & $24,080,550$ & 5 & 7.5 & 5 \\
\hline IRR $>0 \%$ & Scenario 2 & Bituminous high volatiles B & $12,842,960$ & 5 & 4.0 & 7.5 \\
\hline IRR $>0 \%$ & Scenario 2 & Bituminous high volatiles B & $8,412,139$ & 5 & 2.62 & 10 \\
\hline IRR & Price & Rank & GIP area min & GIS min. & Height min. & Perm \\
\hline 10 years & $€ / \mathrm{m}^{3}$ & & $\mathrm{~m}^{3}$ & $\mathrm{~m}^{3} / \mathrm{tp}$ & $\mathrm{m}$ & $\mathrm{mD}$ \\
\hline IRR $>0 \%$ & Scenario 3 & Anthracite & $601,050,528$ & 12 & 78 & 1 \\
\hline IRR $>0 \%$ & Scenario 3 & Anthracite & $130,998,192$ & 12 & 17.0 & 2.5 \\
\hline IRR $>0 \%$ & Scenario 3 & Anthracite & $36,987,725$ & 12 & 4.8 & 5 \\
\hline IRR $>0 \%$ & Scenario 3 & Anthracite & $19,264,440$ & 12 & 2.5 & 7.5 \\
\hline IRR $>0 \%$ & Scenario 3 & Anthracite & $12,791,588$ & 12 & 1.66 & 10 \\
\hline IRR $>0 \%$ & Scenario 3 & Bituminous low volatiles & $400,700,352$ & 8 & 78 & 1 \\
\hline IRR $>0 \%$ & Scenario 3 & Bituminous low volatiles & $87,332,128$ & 8 & 17.0 & 2.5 \\
\hline IRR $>0 \%$ & Scenario 3 & Bituminous low volatiles & $24,658,483$ & 8 & 4.8 & 5 \\
\hline IRR $>0 \%$ & Scenario 3 & Bituminous low volatiles & $12,842,960$ & 8 & 2.5 & 7.5 \\
\hline IRR $>0 \%$ & Scenario 3 & Bituminous low volatiles & $8,527,725$ & 8 & 1.66 & 10 \\
\hline IRR $>0 \%$ & Scenario 3 & Bituminous medium volatiles & $350,612,808$ & 7 & 78 & 1 \\
\hline IRR $>0 \%$ & Scenario 3 & Bituminous medium volatiles & $76,415,612$ & 7 & 17.0 & 2.5 \\
\hline IRR $>0 \%$ & Scenario 3 & Bituminous medium volatiles & $21,576,173$ & 7 & 4.8 & 5 \\
\hline IRR $>0 \%$ & Scenario 3 & Bituminous medium volatiles & $11,237,590$ & 7 & 2.5 & 7.5 \\
\hline IRR $>0 \%$ & Scenario 3 & Bituminous medium volatiles & $7,461,760$ & 7 & 1.66 & 10 \\
\hline IRR $>0 \%$ & Scenario 3 & Bituminous high volatiles A & $300,525,264$ & 6 & 78 & 1 \\
\hline IRR $>0 \%$ & Scenario 3 & Bituminous high volatiles A & $65,499,096$ & 6 & 17.0 & 2.5 \\
\hline IRR $>0 \%$ & Scenario 3 & Bituminous high volatiles A & $18,493,862$ & 6 & 4.8 & 5 \\
\hline IRR $>0 \%$ & Scenario 3 & Bituminous high volatiles A & $9,632,220$ & 6 & 2.5 & 7.5 \\
\hline IRR $>0 \%$ & Scenario 3 & Bituminous high volatiles A & $6,395,794$ & 6 & 1.66 & 10 \\
\hline IRR $>0 \%$ & Scenario 3 & Bituminous high volatiles B & $250,437,720$ & 5 & 78 & 1 \\
\hline IRR $>0 \%$ & Scenario 3 & Bituminous high volatiles B & $54,582,580$ & 5 & 17.0 & 2.5 \\
\hline IRR $>0 \%$ & Scenario 3 & Bituminous high volatiles B & $15,411,552$ & 5 & 4.8 & 5 \\
\hline IRR $>0 \%$ & Scenario 3 & Bituminous high volatiles B & $8,026,850$ & 5 & 2.5 & 7.5 \\
\hline IRR $>0 \%$ & Scenario 3 & Bituminous high volatiles $B$ & $5,329,828$ & 5 & 1.66 & 10 \\
\hline
\end{tabular}


J. A. Gutiérrez et al.

Continued

\begin{tabular}{|c|c|c|c|c|c|c|}
\hline IRR & Price & Rank & GIP area min & GIS min. & Height min. & Perm \\
\hline 10 years & $€ / \mathrm{m}^{3}$ & & $\mathrm{~m}^{3}$ & $\mathrm{~m}^{3} / \mathrm{tp}$ & m & $\mathrm{mD}$ \\
\hline IRR $>0 \%$ & Scenario 4 & Anthracite & $516,286,992$ & 12 & 67 & 1 \\
\hline IRR $>0 \%$ & Scenario 4 & Anthracite & $107,880,864$ & 12 & 14.0 & 2.5 \\
\hline IRR $>0 \%$ & Scenario 4 & Anthracite & $31,593,682$ & 12 & 4.1 & 5 \\
\hline IRR $>0 \%$ & Scenario 4 & Anthracite & $16,952,707$ & 12 & 2.2 & 7.5 \\
\hline IRR $>0 \%$ & Scenario 4 & Anthracite & $10,865,144$ & 12 & 1.41 & 10 \\
\hline IRR $>0 \%$ & Scenario 4 & Bituminous low volatiles & $344,191,328$ & 8 & 67 & 1 \\
\hline IRR $>0 \%$ & Scenario 4 & Bituminous low volatiles & $71,920,576$ & 8 & 14.0 & 2.5 \\
\hline IRR $>0 \%$ & Scenario 4 & Bituminous low volatiles & $21,062,454$ & 8 & 4.1 & 5 \\
\hline IRR $>0 \%$ & Scenario 4 & Bituminous low volatiles & $11,301,805$ & 8 & 2.2 & 7.5 \\
\hline IRR $>0 \%$ & Scenario 4 & Bituminous low volatiles & $7,243,429$ & 8 & 1.41 & 10 \\
\hline IRR $>0 \%$ & Scenario 4 & Bituminous medium volatiles & $301,167,412$ & 7 & 67 & 1 \\
\hline IRR $>0 \%$ & Scenario 4 & Bituminous medium volatiles & $62,930,504$ & 7 & 14.0 & 2.5 \\
\hline IRR $>0 \%$ & Scenario 4 & Bituminous medium volatiles & $18,429,648$ & 7 & 4.1 & 5 \\
\hline IRR $>0 \%$ & Scenario 4 & Bituminous medium volatiles & $9,889,079$ & 7 & 2.2 & 7.5 \\
\hline IRR $>0 \%$ & Scenario 4 & Bituminous medium volatiles & $6,338,001$ & 7 & 1.41 & 10 \\
\hline IRR $>0 \%$ & Scenario 4 & Bituminous high volatiles A & $258,143,496$ & 6 & 67 & 1 \\
\hline IRR $>0 \%$ & Scenario 4 & Bituminous high volatiles A & $53,940,432$ & 6 & 14.0 & 2.5 \\
\hline IRR $>0 \%$ & Scenario 4 & Bituminous high volatiles A & $15,796,841$ & 6 & 4.1 & 5 \\
\hline IRR $>0 \%$ & Scenario 4 & Bituminous high volatiles A & $8,476,354$ & 6 & 2.2 & 7.5 \\
\hline IRR $>0 \%$ & Scenario 4 & Bituminous high volatiles A & $5,432,572$ & 6 & 1.41 & 10 \\
\hline IRR $>0 \%$ & Scenario 4 & Bituminous high volatiles B & $215,119,580$ & 5 & 67 & 1 \\
\hline IRR $>0 \%$ & Scenario 4 & Bituminous high volatiles B & $44,950,360$ & 5 & 14.0 & 2.5 \\
\hline IRR $>0 \%$ & Scenario 4 & Bituminous high volatiles B & $13,164,034$ & 5 & 4.1 & 5 \\
\hline IRR $>0 \%$ & Scenario 4 & Bituminous high volatiles B & $7,063,628$ & 5 & 2.2 & 7.5 \\
\hline IRR $>0 \%$ & Scenario 4 & Bituminous high volatiles B & $4,527,143$ & 5 & 1.41 & 10 \\
\hline IRR & Price & Rank & GIP area min & GIS min. & Height min. & Perm \\
\hline 10 years & $€ / \mathrm{m}^{3}$ & & $\mathrm{~m}^{3}$ & $\mathrm{~m}^{3} / \mathrm{tp}$ & m & $\mathrm{mD}$ \\
\hline IRR $>4 \%$ & Scenario 1 & Anthracite & $847,635,360$ & 12 & 110 & 1 \\
\hline IRR $>4 \%$ & Scenario 1 & Anthracite & $177,232,848$ & 12 & 23.0 & 2.5 \\
\hline IRR $>4 \%$ & Scenario 1 & Anthracite & $50,087,544$ & 12 & 6.5 & 5 \\
\hline IRR $>4 \%$ & Scenario 1 & Anthracite & $26,970,216$ & 12 & 3.5 & 7.5 \\
\hline IRR $>4 \%$ & Scenario 1 & Anthracite & $17,723,285$ & 12 & 2.30 & 10 \\
\hline IRR $>4 \%$ & Scenario 1 & Bituminous low volatiles & $565,090,240$ & 8 & 110 & 1 \\
\hline IRR $>4 \%$ & Scenario 1 & Bituminous low volatiles & $118,155,232$ & 8 & 23.0 & 2.5 \\
\hline IRR $>4 \%$ & Scenario 1 & Bituminous low volatiles & $33,391,696$ & 8 & 6.5 & 5 \\
\hline
\end{tabular}




\section{Continued}

\begin{tabular}{|c|c|c|c|c|c|c|}
\hline IRR $>4 \%$ & Scenario 1 & Bituminous low volatiles & $17,980,144$ & 8 & 3.5 & 7.5 \\
\hline IRR $>4 \%$ & Scenario 1 & Bituminous low volatiles & $11,815,523$ & 8 & 2.30 & 10 \\
\hline $\mathrm{IRR}>4 \%$ & Scenario 1 & Bituminous medium volatiles & $494,453,960$ & 7 & 110 & 1 \\
\hline $\mathrm{IRR}>4 \%$ & Scenario 1 & Bituminous medium volatiles & $103,385,828$ & 7 & 23.0 & 2.5 \\
\hline $\mathrm{IRR}>4 \%$ & Scenario 1 & Bituminous medium volatiles & $29,217,734$ & 7 & 6.5 & 5 \\
\hline $\mathrm{IRR}>4 \%$ & Scenario 1 & Bituminous medium volatiles & $15,732,626$ & 7 & 3.5 & 7.5 \\
\hline $\mathrm{IRR}>4 \%$ & Scenario 1 & Bituminous medium volatiles & $10,338,583$ & 7 & 2.30 & 10 \\
\hline $\mathrm{IRR}>4 \%$ & Scenario 1 & Bituminous high volatiles A & $423,817,680$ & 6 & 110 & 1 \\
\hline IRR $>4 \%$ & Scenario 1 & Bituminous high volatiles A & $88,616,424$ & 6 & 23.0 & 2.5 \\
\hline $\mathrm{IRR}>4 \%$ & Scenario 1 & Bituminous high volatiles A & $25,043,772$ & 6 & 6.5 & 5 \\
\hline $\mathrm{IRR}>4 \%$ & Scenario 1 & Bituminous high volatiles A & $13,485,108$ & 6 & 3.5 & 7.5 \\
\hline $\mathrm{IRR}>4 \%$ & Scenario 1 & Bituminous high volatiles A & $8,861,642$ & 6 & 2.30 & 10 \\
\hline $\mathrm{IRR}>4 \%$ & Scenario 1 & Bituminous high volatiles $B$ & $353,181,400$ & 5 & 110 & 1 \\
\hline $\mathrm{IRR}>4 \%$ & Scenario 1 & Bituminous high volatiles B & $73,847,020$ & 5 & 23.0 & 2.5 \\
\hline $\mathrm{IRR}>4 \%$ & Scenario 1 & Bituminous high volatiles B & $20,869,810$ & 5 & 6.5 & 5 \\
\hline $\mathrm{IRR}>4 \%$ & Scenario 1 & Bituminous high volatiles B & $11,237,590$ & 5 & 3.5 & 7.5 \\
\hline $\mathrm{IRR}>4 \%$ & Scenario 1 & Bituminous high volatiles B & $7,384,702$ & 5 & 2.30 & 10 \\
\hline IRR & Price & Rank & GIP area min & GIS min. & Height min. & Perm \\
\hline 10 years & $€ / \mathrm{m}^{3}$ & & $\mathrm{~m}^{3}$ & $\mathrm{~m}^{3} / \mathrm{tp}$ & $\mathrm{m}$ & $\mathrm{mD}$ \\
\hline $\mathrm{IRR}>4 \%$ & Scenario 2 & Anthracite & $1,078,808,640$ & 12 & 140 & 1 \\
\hline $\mathrm{IRR}>4 \%$ & Scenario 2 & Anthracite & $223,467,504$ & 12 & 29.0 & 2.5 \\
\hline $\mathrm{IRR}>4 \%$ & Scenario 2 & Anthracite & $63,187,363$ & 12 & 8.2 & 5 \\
\hline $\mathrm{IRR}>4 \%$ & Scenario 2 & Anthracite & $33,905,414$ & 12 & 4.4 & 7.5 \\
\hline $\mathrm{IRR}>4 \%$ & Scenario 2 & Anthracite & $22,346,750$ & 12 & 2.90 & 10 \\
\hline $\mathrm{IRR}>4 \%$ & Scenario 2 & Bituminous low volatiles & $719,205,760$ & 8 & 140 & 1 \\
\hline $\mathrm{IRR}>4 \%$ & Scenario 2 & Bituminous low volatiles & $148,978,336$ & 8 & 29.0 & 2.5 \\
\hline $\mathrm{IRR}>4 \%$ & Scenario 2 & Bituminous low volatiles & $42,124,909$ & 8 & 8.2 & 5 \\
\hline $\mathrm{IRR}>4 \%$ & Scenario 2 & Bituminous low volatiles & $22,603,610$ & 8 & 4.4 & 7.5 \\
\hline $\mathrm{IRR}>4 \%$ & Scenario 2 & Bituminous low volatiles & $14,897,834$ & 8 & 2.90 & 10 \\
\hline $\mathrm{IRR}>4 \%$ & Scenario 2 & Bituminous medium volatiles & $629,305,040$ & 7 & 140 & 1 \\
\hline $\mathrm{IRR}>4 \%$ & Scenario 2 & Bituminous medium volatiles & $130,356,044$ & 7 & 29.0 & 2.5 \\
\hline $\mathrm{IRR}>4 \%$ & Scenario 2 & Bituminous medium volatiles & $36,859,295$ & 7 & 8.2 & 5 \\
\hline $\mathrm{IRR}>4 \%$ & Scenario 2 & Bituminous medium volatiles & $19,778,158$ & 7 & 4.4 & 7.5 \\
\hline $\mathrm{IRR}>4 \%$ & Scenario 2 & Bituminous medium volatiles & $13,035,604$ & 7 & 2.90 & 10 \\
\hline IRR $>4 \%$ & Scenario 2 & Bituminous high volatiles A & $539,404,320$ & 6 & 140 & 1 \\
\hline $\mathrm{IRR}>4 \%$ & Scenario 2 & Bituminous high volatiles A & $111,733,752$ & 6 & 29.0 & 2.5 \\
\hline IRR $>4 \%$ & Scenario 2 & Bituminous high volatiles A & $31,593,682$ & 6 & 8.2 & 5 \\
\hline
\end{tabular}




\begin{tabular}{|c|c|c|c|c|c|c|}
\hline $\mathrm{IRR}>4 \%$ & Scenario 2 & Bituminous high volatiles A & $16,952,707$ & 6 & 4.4 & 7.5 \\
\hline $\mathrm{IRR}>4 \%$ & Scenario 2 & Bituminous high volatiles A & $11,173,375$ & 6 & 2.90 & 10 \\
\hline IRR $>4 \%$ & Scenario 2 & Bituminous high volatiles $B$ & $449,503,600$ & 5 & 140 & 1 \\
\hline $\mathrm{IRR}>4 \%$ & Scenario 2 & Bituminous high volatiles B & $93,111,460$ & 5 & 29.0 & 2.5 \\
\hline $\mathrm{IRR}>4 \%$ & Scenario 2 & Bituminous high volatiles B & $26,328,068$ & 5 & 8.2 & 5 \\
\hline $\mathrm{IRR}>4 \%$ & Scenario 2 & Bituminous high volatiles B & $14,127,256$ & 5 & 4.4 & 7.5 \\
\hline IRR $>4 \%$ & Scenario 2 & Bituminous high volatiles B & $9,311,146$ & 5 & 2.90 & 10 \\
\hline IRR & Price & Rank & GIP area min & GIS min. & Height min. & Perm \\
\hline 10 years & $€ / \mathrm{m}^{3}$ & & $\mathrm{~m}^{3}$ & $\mathrm{~m}^{3} / \mathrm{tp}$ & $\mathrm{m}$ & $\mathrm{mD}$ \\
\hline $\mathrm{IRR}>4 \%$ & Scenario 3 & Anthracite & $662,696,736$ & 12 & 86 & 1 \\
\hline $\mathrm{IRR}>4 \%$ & Scenario 3 & Anthracite & $138,703,968$ & 12 & 18.0 & 2.5 \\
\hline $\mathrm{IRR}>4 \%$ & Scenario 3 & Anthracite & $40,070,035$ & 12 & 5.2 & 5 \\
\hline $\mathrm{IRR}>4 \%$ & Scenario 3 & Anthracite & $21,576,173$ & 12 & 2.8 & 7.5 \\
\hline $\mathrm{IRR}>4 \%$ & Scenario 3 & Anthracite & $14,101,570$ & 12 & 1.83 & 10 \\
\hline $\mathrm{IRR}>4 \%$ & Scenario 3 & Bituminous low volatiles & $441,797,824$ & 8 & 86 & 1 \\
\hline $\mathrm{IRR}>4 \%$ & Scenario 3 & Bituminous low volatiles & $92,469,312$ & 8 & 18.0 & 2.5 \\
\hline $\mathrm{IRR}>4 \%$ & Scenario 3 & Bituminous low volatiles & $26,713,357$ & 8 & 5.2 & 5 \\
\hline $\mathrm{IRR}>4 \%$ & Scenario 3 & Bituminous low volatiles & $14,384,115$ & 8 & 2.8 & 7.5 \\
\hline $\mathrm{IRR}>4 \%$ & Scenario 3 & Bituminous low volatiles & $9,401,047$ & 8 & 1.83 & 10 \\
\hline $\mathrm{IRR}>4 \%$ & Scenario 3 & Bituminous medium volatiles & $386,573,096$ & 7 & 86 & 1 \\
\hline $\mathrm{IRR}>4 \%$ & Scenario 3 & Bituminous medium volatiles & $80,910,648$ & 7 & 18.0 & 2.5 \\
\hline IRR $>4 \%$ & Scenario 3 & Bituminous medium volatiles & $23,374,187$ & 7 & 5.2 & 5 \\
\hline $\mathrm{IRR}>4 \%$ & Scenario 3 & Bituminous medium volatiles & $12,586,101$ & 7 & 2.8 & 7.5 \\
\hline IRR $>4 \%$ & Scenario 3 & Bituminous medium volatiles & $8,225,916$ & 7 & 1.83 & 10 \\
\hline $\mathrm{IRR}>4 \%$ & Scenario 3 & Bituminous high volatiles A & $331,348,368$ & 6 & 86 & 1 \\
\hline $\mathrm{IRR}>4 \%$ & Scenario 3 & Bituminous high volatiles A & $69,351,984$ & 6 & 18.0 & 2.5 \\
\hline $\mathrm{IRR}>4 \%$ & Scenario 3 & Bituminous high volatiles A & $20,035,018$ & 6 & 5.2 & 5 \\
\hline $\mathrm{IRR}>4 \%$ & Scenario 3 & Bituminous high volatiles A & $10,788,086$ & 6 & 2.8 & 7.5 \\
\hline $\mathrm{IRR}>4 \%$ & Scenario 3 & Bituminous high volatiles A & $7,050,785$ & 6 & 1.83 & 10 \\
\hline $\mathrm{IRR}>4 \%$ & Scenario 3 & Bituminous high volatiles B & $276,123,640$ & 5 & 86 & 1 \\
\hline $\mathrm{IRR}>4 \%$ & Scenario 3 & Bituminous high volatiles B & $57,793,320$ & 5 & 18.0 & 2.5 \\
\hline $\mathrm{IRR}>4 \%$ & Scenario 3 & Bituminous high volatiles $B$ & $16,695,848$ & 5 & 5.2 & 5 \\
\hline IRR $>4 \%$ & Scenario 3 & Bituminous high volatiles $B$ & $8,990,072$ & 5 & 2.8 & 7.5 \\
\hline IRR $>4 \%$ & Scenario 3 & Bituminous high volatiles $B$ & $5,875,654$ & 5 & 1.83 & 10 \\
\hline IRR & Price & Rank & GIP area min & GIS min. & Height min. & Perm \\
\hline 10 years & $€ / \mathrm{m}^{3}$ & & $\mathrm{~m}^{3}$ & $\mathrm{~m}^{3} / \mathrm{tp}$ & $\mathrm{m}$ & $\mathrm{mD}$ \\
\hline IRR $>4 \%$ & Scenario 4 & Anthracite & $547,110,096$ & 12 & 71 & 1 \\
\hline
\end{tabular}




\section{Continued}

\begin{tabular}{|c|c|c|c|c|c|c|}
\hline $\mathrm{IRR}>4 \%$ & Scenario 4 & Anthracite & $115,586,640$ & 12 & 15.0 & 2.5 \\
\hline IRR $>4 \%$ & Scenario 4 & Anthracite & $33,134,837$ & 12 & 4.3 & 5 \\
\hline $\mathrm{IRR}>4 \%$ & Scenario 4 & Anthracite & $17,723,285$ & 12 & 2.3 & 7.5 \\
\hline $\mathrm{IRR}>4 \%$ & Scenario 4 & Anthracite & $11,558,664$ & 12 & 1.50 & 10 \\
\hline $\mathrm{IRR}>4 \%$ & Scenario 4 & Bituminous low volatiles & $364,740,064$ & 8 & 71 & 1 \\
\hline $\mathrm{IRR}>4 \%$ & Scenario 4 & Bituminous low volatiles & $77,057,760$ & 8 & 15.0 & 2.5 \\
\hline IRR $>4 \%$ & Scenario 4 & Bituminous low volatiles & $22,089,891$ & 8 & 4.3 & 5 \\
\hline $\mathrm{IRR}>4 \%$ & Scenario 4 & Bituminous low volatiles & $11,815,523$ & 8 & 2.3 & 7.5 \\
\hline $\mathrm{IRR}>4 \%$ & Scenario 4 & Bituminous low volatiles & $7,705,776$ & 8 & 1.50 & 10 \\
\hline $\mathrm{IRR}>4 \%$ & Scenario 4 & Bituminous medium volatiles & $319,147,556$ & 7 & 71 & 1 \\
\hline $\mathrm{IRR}>4 \%$ & Scenario 4 & Bituminous medium volatiles & $67,425,540$ & 7 & 15.0 & 2.5 \\
\hline $\mathrm{IRR}>4 \%$ & Scenario 4 & Bituminous medium volatiles & $19,328,655$ & 7 & 4.3 & 5 \\
\hline $\mathrm{IRR}>4 \%$ & Scenario 4 & Bituminous medium volatiles & $10,338,583$ & 7 & 2.3 & 7.5 \\
\hline $\mathrm{IRR}>4 \%$ & Scenario 4 & Bituminous medium volatiles & $6,742,554$ & 7 & 1.50 & 10 \\
\hline $\mathrm{IRR}>4 \%$ & Scenario 4 & Bituminous high volatiles A & $273,555,048$ & 6 & 71 & 1 \\
\hline $\mathrm{IRR}>4 \%$ & Scenario 4 & Bituminous high volatiles A & $57,793,320$ & 6 & 15.0 & 2.5 \\
\hline $\mathrm{IRR}>4 \%$ & Scenario 4 & Bituminous high volatiles A & $16,567,418$ & 6 & 4.3 & 5 \\
\hline $\mathrm{IRR}>4 \%$ & Scenario 4 & Bituminous high volatiles A & $8,861,642$ & 6 & 2.3 & 7.5 \\
\hline $\mathrm{IRR}>4 \%$ & Scenario 4 & Bituminous high volatiles A & $5,779,332$ & 6 & 1.50 & 10 \\
\hline $\mathrm{IRR}>4 \%$ & Scenario 4 & Bituminous high volatiles B & $227,962,540$ & 5 & 71 & 1 \\
\hline $\mathrm{IRR}>4 \%$ & Scenario 4 & Bituminous high volatiles B & $48,161,100$ & 5 & 15.0 & 2.5 \\
\hline $\mathrm{IRR}>4 \%$ & Scenario 4 & Bituminous high volatiles B & $13,806,182$ & 5 & 4.3 & 5 \\
\hline $\mathrm{IRR}>4 \%$ & Scenario 4 & Bituminous high volatiles B & $7,384,702$ & 5 & 2.3 & 7.5 \\
\hline $\mathrm{IRR}>4 \%$ & Scenario 4 & Bituminous high volatiles B & $4,816,110$ & 5 & 1.50 & 10 \\
\hline IRR & Price & Rank & GIP area min & GIS min. & Height min. & Perm \\
\hline 10 years & $€ / \mathrm{m}^{3}$ & & $\mathrm{~m}^{3}$ & $\mathrm{~m}^{3} / \mathrm{tp}$ & $\mathrm{m}$ & $\mathrm{mD}$ \\
\hline $\mathrm{IRR}>8 \%$ & Scenario 1 & Anthracite & $924,693,120$ & 12 & 120 & 1 \\
\hline IRR $>8 \%$ & Scenario 1 & Anthracite & $192,644,400$ & 12 & 25 & 2,5 \\
\hline IRR $>8 \%$ & Scenario 1 & Anthracite & $55,481,587$ & 12 & 7.2 & 5 \\
\hline IRR $>8 \%$ & Scenario 1 & Anthracite & $30,052,526$ & 12 & 3.9 & 7.5 \\
\hline $\mathrm{IRR}>8 \%$ & Scenario 1 & Anthracite & $19,495,613$ & 12 & 2.53 & 10 \\
\hline IRR $>8 \%$ & Scenario 1 & Bituminous low volatiles & $616,462,080$ & 8 & 120 & 1 \\
\hline $\mathrm{IRR}>8 \%$ & Scenario 1 & Bituminous low volatiles & $128,429,600$ & 8 & 25 & 2.5 \\
\hline IRR $>8 \%$ & Scenario 1 & Bituminous low volatiles & $36,987,725$ & 8 & 7.2 & 5 \\
\hline $\mathrm{IRR}>8 \%$ & Scenario 1 & Bituminous low volatiles & $20,035,018$ & 8 & 3.9 & 7.5 \\
\hline $\mathrm{IRR}>8 \%$ & Scenario 1 & Bituminous low volatiles & $12,997,076$ & 8 & 2.53 & 10 \\
\hline $\mathrm{IRR}>8 \%$ & Scenario 1 & Bituminous medium volatiles & $539,404,320$ & 7 & 120 & 1 \\
\hline
\end{tabular}




\section{Continued}

\begin{tabular}{|c|c|c|c|c|c|c|}
\hline $\mathrm{IRR}>8 \%$ & Scenario 1 & Bituminous medium volatiles & $112,375,900$ & 7 & 25 & 2.5 \\
\hline IRR $>8 \%$ & Scenario 1 & Bituminous medium volatiles & $32,364,259$ & 7 & 7.2 & 5 \\
\hline $\mathrm{IRR}>8 \%$ & Scenario 1 & Bituminous medium volatiles & $17,530,640$ & 7 & 3.9 & 7.5 \\
\hline IRR $>8 \%$ & Scenario 1 & Bituminous medium volatiles & $11,372,441$ & 7 & 2.53 & 10 \\
\hline $\mathrm{IRR}>8 \%$ & Scenario 1 & Bituminous high volatiles A & $462,346,560$ & 6 & 120 & 1 \\
\hline IRR $>8 \%$ & Scenario 1 & Bituminous high volatiles A & $96,322,200$ & 6 & 25 & 2.5 \\
\hline IRR $>8 \%$ & Scenario 1 & Bituminous high volatiles A & $27,740,794$ & 6 & 7.2 & 5 \\
\hline IRR $>8 \%$ & Scenario 1 & Bituminous high volatiles A & $15,026,263$ & 6 & 3.9 & 7.5 \\
\hline $\mathrm{IRR}>8 \%$ & Scenario 1 & Bituminous high volatiles A & $9,747,807$ & 6 & 2.53 & 10 \\
\hline $\mathrm{IRR}>8 \%$ & Scenario 1 & Bituminous high volatiles $B$ & $385,288,800$ & 5 & 120 & 1 \\
\hline $\mathrm{IRR}>8 \%$ & Scenario 1 & Bituminous high volatiles $B$ & $80,268,500$ & 5 & 25 & 2.5 \\
\hline $\mathrm{IRR}>8 \%$ & Scenario 1 & Bituminous high volatiles B & $23,117,328$ & 5 & 7.2 & 5 \\
\hline $\mathrm{IRR}>8 \%$ & Scenario 1 & Bituminous high volatiles B & $12,521,886$ & 5 & 3.9 & 7.5 \\
\hline $\mathrm{IRR}>8 \%$ & Scenario 1 & Bituminous high volatiles B & $8,123,172$ & 5 & 2.53 & 10 \\
\hline IRR & Price & Rank & GIP area min & GIS min. & Height min. & Perm \\
\hline 10 years & $€ / \mathrm{m}^{3}$ & & $\mathrm{~m}^{3}$ & $\mathrm{~m}^{3} / \mathrm{tp}$ & $\mathrm{m}$ & $\mathrm{mD}$ \\
\hline IRR $>8 \%$ & Scenario 2 & Anthracite & $1,155,866,400$ & 12 & 150 & 1 \\
\hline $\mathrm{IRR}>8 \%$ & Scenario 2 & Anthracite & $238,879,056$ & 12 & 31 & 2.5 \\
\hline $\mathrm{IRR}>8 \%$ & Scenario 2 & Anthracite & $69,351,984$ & 12 & 9.0 & 5 \\
\hline IRR $>8 \%$ & Scenario 2 & Anthracite & $36,987,725$ & 12 & 4.8 & 7.5 \\
\hline IRR $>8 \%$ & Scenario 2 & Anthracite & $24,273,194$ & 12 & 3.15 & 10 \\
\hline $\mathrm{IRR}>8 \%$ & Scenario 2 & Bituminous low volatiles & $770,577,600$ & 8 & 150 & 1 \\
\hline $\mathrm{IRR}>8 \%$ & Scenario 2 & Bituminous low volatiles & $159,252,704$ & 8 & 31 & 2.5 \\
\hline IRR $>8 \%$ & Scenario 2 & Bituminous low volatiles & $46,234,656$ & 8 & 9.0 & 5 \\
\hline IRR $>8 \%$ & Scenario 2 & Bituminous low volatiles & $24,658,483$ & 8 & 4.8 & 7.5 \\
\hline IRR $>8 \%$ & Scenario 2 & Bituminous low volatiles & $16,182,130$ & 8 & 3.15 & 10 \\
\hline $\mathrm{IRR}>8 \%$ & Scenario 2 & Bituminous medium volatiles & $674,255,400$ & 7 & 150 & 1 \\
\hline $\mathrm{IRR}>8 \%$ & Scenario 2 & Bituminous medium volatiles & $139,346,116$ & 7 & 31 & 2.5 \\
\hline $\mathrm{IRR}>8 \%$ & Scenario 2 & Bituminous medium volatiles & $40,455,324$ & 7 & 9.0 & 5 \\
\hline IRR $>8 \%$ & Scenario 2 & Bituminous medium volatiles & $21,576,173$ & 7 & 4.8 & 7.5 \\
\hline $\mathrm{IRR}>8 \%$ & Scenario 2 & Bituminous medium volatiles & $14,159,363$ & 7 & 3.15 & 10 \\
\hline IRR $>8 \%$ & Scenario 2 & Bituminous high volatiles A & $577,933,200$ & 6 & 150 & 1 \\
\hline IRR $>8 \%$ & Scenario 2 & Bituminous high volatiles A & $119,439,528$ & 6 & 31 & 2.5 \\
\hline $\mathrm{IRR}>8 \%$ & Scenario 2 & Bituminous high volatiles A & $34,675,992$ & 6 & 9.0 & 5 \\
\hline IRR $>8 \%$ & Scenario 2 & Bituminous high volatiles A & $18,493,862$ & 6 & 4.8 & 7.5 \\
\hline $\mathrm{IRR}>8 \%$ & Scenario 2 & Bituminous high volatiles A & $12,136,597$ & 6 & 3.15 & 10 \\
\hline IRR $>8 \%$ & Scenario 2 & Bituminous high volatiles B & $481,611,000$ & 5 & 150 & 1 \\
\hline
\end{tabular}




\section{Continued}

\begin{tabular}{|c|c|c|c|c|c|c|}
\hline IRR $>8 \%$ & Scenario 2 & Bituminous high volatiles B & $99,532,940$ & 5 & 31 & 2.5 \\
\hline IRR $>8 \%$ & Scenario 2 & Bituminous high volatiles B & $28,896,660$ & 5 & 9.0 & 5 \\
\hline IRR $>8 \%$ & Scenario 2 & Bituminous high volatiles B & $15,411,552$ & 5 & 4.8 & 7.5 \\
\hline IRR $>8 \%$ & Scenario 2 & Bituminous high volatiles B & $10,113,831$ & 5 & 3.15 & 10 \\
\hline IRR & Price & Rank & GIP area min & GIS min, & Height min, & Perm \\
\hline 10 years & $€ / \mathrm{m}^{3}$ & & $\mathrm{~m}^{3}$ & $\mathrm{~m}^{3} / \mathrm{tp}$ & m & $\mathrm{mD}$ \\
\hline IRR $>8 \%$ & Scenario 3 & Anthracite & $724,342,944$ & 12 & 94 & 1 \\
\hline IRR $>8 \%$ & Scenario 3 & Anthracite & $154,115,520$ & 12 & 20 & 2.5 \\
\hline IRR $>8 \%$ & Scenario 3 & Anthracite & $43,922,923$ & 12 & 5.7 & 5 \\
\hline IRR $>8 \%$ & Scenario 3 & Anthracite & $23,117,328$ & 12 & 3.0 & 7.5 \\
\hline IRR $>8 \%$ & Scenario 3 & Anthracite & $15,411,552$ & 12 & 2.00 & 10 \\
\hline IRR $>8 \%$ & Scenario 3 & Bituminous low volatiles & $482,895,296$ & 8 & 94 & 1 \\
\hline IRR $>8 \%$ & Scenario 3 & Bituminous low volatiles & $102,743,680$ & 8 & 20 & 2.5 \\
\hline IRR $>8 \%$ & Scenario 3 & Bituminous low volatiles & $29,281,949$ & 8 & 5.7 & 5 \\
\hline IRR $>8 \%$ & Scenario 3 & Bituminous low volatiles & $15,411,552$ & 8 & 3.0 & 7.5 \\
\hline IRR $>8 \%$ & Scenario 3 & Bituminous low volatiles & $10,274,368$ & 8 & 2.00 & 10 \\
\hline IRR $>8 \%$ & Scenario 3 & Bituminous medium volatiles & $422,533,384$ & 7 & 94 & 1 \\
\hline IRR $>8 \%$ & Scenario 3 & Bituminous medium volatiles & $89,900,720$ & 7 & 20 & 2.5 \\
\hline IRR $>8 \%$ & Scenario 3 & Bituminous medium volatiles & $25,621,705$ & 7 & 5.7 & 5 \\
\hline IRR $>8 \%$ & Scenario 3 & Bituminous medium volatiles & $13,485,108$ & 7 & 3.0 & 7.5 \\
\hline IRR $>8 \%$ & Scenario 3 & Bituminous medium volatiles & $8,990,072$ & 7 & 2.00 & 10 \\
\hline IRR $>8 \%$ & Scenario 3 & Bituminous high volatiles A & $362,171,472$ & 6 & 94 & 1 \\
\hline IRR $>8 \%$ & Scenario 3 & Bituminous high volatiles A & $77,057,760$ & 6 & 20 & 2.5 \\
\hline IRR $>8 \%$ & Scenario 3 & Bituminous high volatiles A & $21,961,462$ & 6 & 5.7 & 5 \\
\hline IRR $>8 \%$ & Scenario 3 & Bituminous high volatiles A & $11,558,664$ & 6 & 3.0 & 7.5 \\
\hline IRR $>8 \%$ & Scenario 3 & Bituminous high volatiles A & $7,705,776$ & 6 & 2.00 & 10 \\
\hline IRR $>8 \%$ & Scenario 3 & Bituminous high volatiles B & $301,809,560$ & 5 & 94 & 1 \\
\hline IRR $>8 \%$ & Scenario 3 & Bituminous high volatiles B & $64,214,800$ & 5 & 20 & 2.5 \\
\hline IRR $>8 \%$ & Scenario 3 & Bituminous high volatiles B & $18,301,218$ & 5 & 5.7 & 5 \\
\hline IRR $>8 \%$ & Scenario 3 & Bituminous high volatiles B & $9,632,220$ & 5 & 3.0 & 7.5 \\
\hline IRR $>8 \%$ & Scenario 3 & Bituminous high volatiles B & $6,421,480$ & 5 & 2.00 & 10 \\
\hline IRR & Price & Rank & GIP area min & GIS min, & Height min, & Perm \\
\hline 10 years & $€ / \mathrm{m}^{3}$ & & $\mathrm{~m}^{3}$ & $\mathrm{~m}^{3} / \mathrm{tp}$ & $\mathrm{m}$ & $\mathrm{mD}$ \\
\hline IRR $>8 \%$ & Scenario 4 & Anthracite & $601,050,528$ & 12 & 78 & 1 \\
\hline IRR $>8 \%$ & Scenario 4 & Anthracite & $130,998,192$ & 12 & 17.0 & 2.5 \\
\hline IRR $>8 \%$ & Scenario 4 & Anthracite & $36,987,725$ & 12 & 4.8 & 5 \\
\hline IRR $>8 \%$ & Scenario 4 & Anthracite & $19,264,440$ & 12 & 2.5 & 7.5 \\
\hline
\end{tabular}




\section{Continued}

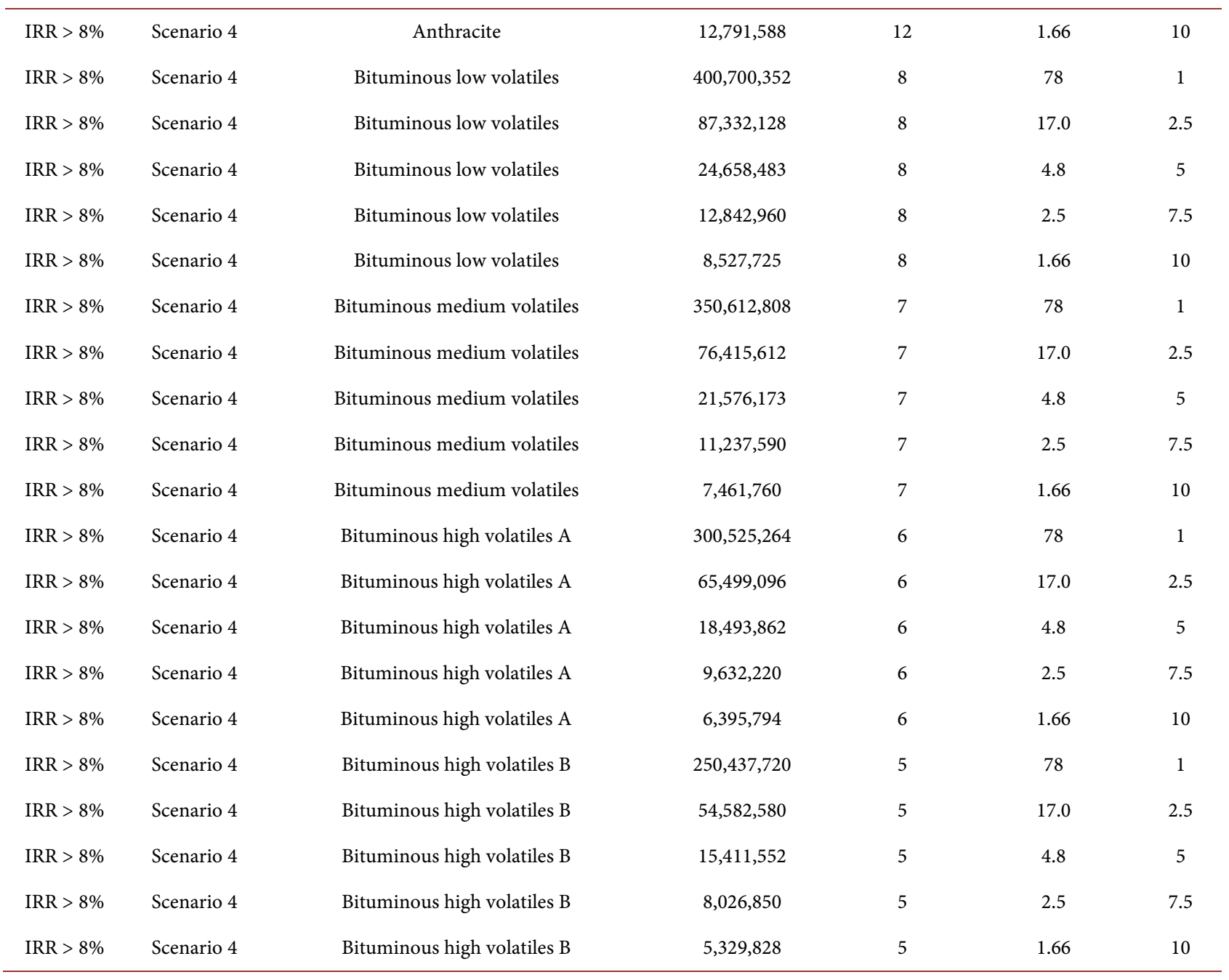

the rank of the coal in our reserve.

Subsequently, we must verify that the permeability, height and gas and GIP values of the area affected by the well are greater than or equal to those defined in the corresponding table. The minimum GIP divided by the height will give a layer surface. This surface corrected with the dip will give us a surface area to drain, which will determine the minimum spacing of the wells.

As a remark it should be added that these tables are used to determine profitability for vertical drilling and without multilayer drilling. Summary tables for project viability are shown below.

\section{Conclusions}

The economic viability was calculated with three different profitability objectives for positive evaluation. The first one only contemplates the recovery of the investment (IRR > 0\%), while the second and third raise the possibility of obtaining more return than an investment of $4 \%$ and $8 \%$. For these projections, four possible scenarios were considered of natural gas price in the wholesale market: 
the current price (scenario 1), price reduction by $25 \%$ (scenario 2), price increase by $25 \%$ (scenario 3 ) and price increase $50 \%$ (scenario 4 ).

For the estimation of the economic viability, in a first stage, the annual production of each well was determined and, after this, it was the annual investment and operation costs. Next, revenues were defined for each of the proposed scenarios and profitability was analyzed for each case.

Recovering the results and the methodology followed, we obtained the possibility of carrying out a sensitivity analysis of the technical parameters of the reserve to economic profitability, which resulted in the development of user-friendly tables, through which we can determine the economic viability of any CBM reservation. These tables are based on the quantification of the following factors:

- IRR.

- Price of natural gas.

- Coal rank.

- Gas in place (GIP).

- Gas in situ (GIS) or amount of gas.

- Height.

- Permeability.

For the use of these tables, the IRR and the gas price scenarios are data to be chosen by the designer, therefore subjective. The minimum gas quantity is only a function of the coal rank. Height and permeability are intimately linked. When the permeability is higher, the height needed to obtain profitability of well is reduced. The GIP is a function of GIS, height and spacing. From the above, it is clear that the tables will help to design the well spacing.

Applying these tables to the autonomous community of Castilla y León, it will be possible to know the amount of extractable gas in a profitable manner for 10 or 30 years of production. It will be carried out for gas prices corresponding to scenario 1 , with returns of $0 \%, 4 \%$ and $8 \%$.

Regarding the main geological and geomorphological factors and their relationship with the economic viability of $\mathrm{CBM}$ farms, the following conclusions can be drawn:

- The amount of gas or gas in situ minimum required for viable production is dependent on the rank of coal. This is because for a final downhole pressure each coal can store a given adsorbed gas.

- If the permeability is considered independent of the rank, the key factor to develop a project of this type regarding permeability is the height. Thus a layer with low height but high permeability can be exploitable in a cost-effective manner. The same statement can be established in the opposite direction, since height is usually a more familiar property in coal deposits. It can be inferred that for a given height, the CBM exploitation goes to depend very directly on the permeability obtained in drilling tests.

- The greater the depth, the greater the cost of drilling, but the greater will be the gas adsorbed due to the pressure. The optimum depth to carry out a pro- 
ject of this type will vary between 500 and $1500 \mathrm{~m}$. Above $1500 \mathrm{~m}$ drilling costs increase, as well as the technical complexity of it.

- Regarding the variation in operating time, permeability becomes a key factor once it is shortened. A CBM farm will be more sensitive to exploitation times the lower its permeability. Permeabilities above $15-20 \mathrm{mD}$ present low sensitivity to the reduction of production times.

- The permeability, confirmed as a key factor, will depend exponentially on the width of the cleats network, while it will depend on the spacing in a linear manner, so the width of the cleats will be a crucial factor in the development of the project.

- The expected production time will define very clearly the spacing of the wells. The greater the period of exploitation, the greater the spacing of the wells may be, a circumstance that will increase profitability due to the fact that the highest cost of a well of this type is drilling. The annual fixed operating and maintenance costs for more than 10 years are shown as low in the economic set of the operation, of the order of $€ 50,000 /$ well per year. The spacing will be in the range of 500 and $800 \mathrm{~m}$.

Regarding purely economic factors, the viability of a project of this type will depend more on the evolution of gas prices than on the required profitability. This high sensitivity to gas prices justifies the realization of the production analysis for the 4 different scenarios proposed.

\section{Conflicts of Interest}

The authors declare no conflicts of interest regarding the publication of this paper.

\section{References}

[1] Creedy, D. and Tilley, H. (2003) Coalbed Methane Extraction and Utilization. Proceedings of the Institution of Mechanical Engineers, Part A: Journal of Power and Energy, 217, 19-25.

[2] Eddy, G.E. (1982) Relationship of Methane Content of Coal Rank and Depth: Theoretical vs. Observed. Proceedings of the SPE Unconventional Gas Recovery Symposium, 16-18 May 1982, Pittsburgh, Pennsylvania. https://doi.org/10.2118/10800-MS

[3] Gray, I. (1987) Reservoir Engineering in Coal Seams, the Physical Process of Gas Storage and Movement in Coal Seams. SPE 12514. Reservoir Engineering, 28-40.

[4] Laubach, S.E. (1997) Characteristics and Origins of Coal Cleat: A Review. International Journal of Coal Geology, 35, 175-207.

[5] Cardott, B.J. (2001) Coalbed Methane Activity in Oklahoma 2001. Oklahoma Coalbed-Methane Workshop: Oklahoma Geological Survey, Open-File Report of 2-2001, 93-139.

[6] Weniger, P.R.S. (2016) Characterizing Coal Cleats from Optical Measurements for CBM Evaluation. International Journal of Coal Geology, 154-155, 176-192. https://doi.org/10.1016/j.coal.2015.12.005

[7] Gas Research Institute. (1991) Coalbed Methane of Western North America: 
Guidebook for the Rocky Mountain. Association of Geologists Fall Conference and Field Trip, 17-20 September 1991, Glenwood Springs, Colorado.

[8] Arenas, G. (2004) Development of Gas Production Type Curves for Coalbed Methane Reservoirs. Department of Petroleum and Natural Gas Engineering of West Virginia.

[9] US DOE. (2006) The Economics of Powder River Basin Coalbed Methane Development. 